\title{
Dynamic behavior analysis of a mechanical system with two unstable modes coupled to a single nonlinear energy sink
}

\author{
Baptiste Bergeot ${ }^{\mathrm{a}, *}$, Sergio Bellizzi ${ }^{\mathrm{b}}$, Sébastien Berger ${ }^{\mathrm{a}}$ \\ ${ }^{a}$ INSA CVL, Univ. Orléans, Univ. Tours, LaMé EA 7494, F-41034, 3 Rue de la Chocolaterie, CS 23410, 41034 Blois \\ Cedex, France \\ ${ }^{b}$ Aix Marseille Univ, CNRS, Centrale Marseille, LMA UMR 7031, Marseille, France
}

\begin{abstract}
This paper investigates a problem of passive mitigation of vibratory instabilities caused by two unstable modes by means of a single nonlinear energy sink (NES). For this purpose, a linear four-degree-of-freedom (DOF) primary structure having two unstable modes (reproducing the typical dynamic behavior of a friction system) and undergoing, as it is linear, unbounded motions when it is unstable, is coupled to a NES. In this work, the NES involves an essentially cubic restoring force and a linear damping force. We are interested in characterizing analytically the response regimes resulting from the coupling of the two unstable linear modes of the primary structure and the nonlinear mode of the NES. To this end, from a suitable rescaling of the governing equations of the coupled system in which the dynamics of the primary structure is reduced to its unstable modal coordinates, the complexification-averaging method is applied. The resulting averaged system appears to be a fast-slow system with four fast variables and two slow ones related to the two unstable modes of the primary structure. The critical manifold of the averaged dynamics is obtained through the geometric singular perturbation theory and appears as a two-dimensional parametric surface (with respect to two of the four fast variables) which evolves in the whole six-dimensional variable space. The asymptotic analysis reveals that the NES attachment can produce some bounded responses and suggests that the system may have simultaneous stable attractors. Numerical simulations complement the study, highlighting a possible competition between stable attractors and allowing us to investigate their basins of attraction. In each considered situation, a good agreement has been observed between theoretical results and numerical simulations, which validates the proposed asymptotic analysis.
\end{abstract}

Keywords: Nonlinear energy sink, Multi-instabilities, Relaxation oscillations, Multiple-scale analysis

\section{Introduction}

A nonlinear energy sink (NES) is a vibration absorber used for passive shock and vibration mitigation of undesirable oscillations of a primary structure induced by external, parametric or self-excitations. A NES is classically defined as a nonlinear dynamical attachment consisting of a light mass (compared to the total mass of the primary structure), an essentially nonlinear spring and a viscous linear damper. Properly designed, a NES can be tuned to any frequency to perform an energy transfer from the primary structure towards itself. This phenomenon, called targeted energy transfer (TET), is realized through nonlinear mode bifurcations and it can be described as resonance captures. Reviews of these concepts can be found in [1] and in a part of [2].

The use of NES to control or suppress dynamic instabilities has been widely studied. The first work reported in this field concerns the suppression of limit cycle oscillations of the Van der Pol oscillator [3].

\footnotetext{
* Corresponding author

Email addresses: baptiste.bergeot@insa-cvl.fr (Baptiste Bergeot), bellizzi@lma.cnrs-mrs.fr (Sergio Bellizzi), sebastien.berger@insa-cvl.fr (Sébastien Berger)
} 
Bifurcations of self-excitation regimes in a Van der Pol (respectively Van der Pol-Duffing) oscillator coupled with a NES have been considered in [4] (respectively [5]). Numerous papers [6, 7, 8, 9, 10, 11, 12, 13] have been dedicated to the suppression of aeroelastic instabilities. The mitigation, by means of a NES, of vortexinduced vibrations resulting from the nonlinear interaction of a laminar flow and a rigid circular cylinder has been described in $[14,15]$. The passive suppression of helicopter ground resonance instabilities has been considered in $[16,17]$. Chatter control in machine tool vibrations has been studied in $[18,19]$. Mitigation of friction-induced vibrations due to mode coupling instabilities in a friction system has been investigated in [20]. A network of parallel NES coupled to a Van der Pol oscillator has been considered in [21]. The prediction of the steady-state regimes of a multi-degree-of-freedom dynamical system having one unstable mode coupled to a set of NES has been performed in [22].

The presence of a NES modifies the position of the bifurcation point and the amplitude of the bifurcated branches. It has been shown that NES can mitigate limit cycle oscillations (LCO). Several possible scenarios have been identified including complete suppression, mitigation through periodic response and mitigation through strongly modulated response (SMR). The results have been obtained under the main assumption that the primary system has only one unstable mode. This assumption is the common thread in all previously referenced works.

Mitigations involving two simultaneous resonances have been considered in the cases of external excitations or transient regimes. TET by means of a multi-resonance capture in a 2-DOF system under two different harmonic excitations has been investigated numerically [23, 24, 25] and experimentally [26]. It has been shown that two resonances of the system are possible simultaneously resulting in vibration reduction around the two resonance frequencies. Regarding transient responses, multi-frequency TET cascades have been studied in $[27,28,29]$. Moreover, it has been shown in [30, 31] that a bistable NES is able to efficiently mitigate oscillations of the primary system for more than one vibration mode.

In this paper, we consider the case of a primary system having two unstable modes and we investigate the mitigation of the two instabilities by means of a single NES. To the knowledge of the authors, this case has never been considered in the past. As for the primary system, the retained model, a simple 4-DOF friction system involving two unstable modes, has been introduced in [32] and used in [33] to illustrate different stability behaviors characterized by mode coupling having coalescence patterns. Based on the procedure presented in [22], we provide an analytic framework for characterizing the response regimes.

The paper is organized as follows. In Sect. 2, the system under study is presented. It consists in two 2-DOF Hulten systems connected by one spring and one damper resulting in a 4-DOF system on which one ungrounded NES is fixed along one of the DOF. In Sect. 3, applying a complexification-averaging method, the analyses of the critical manifold, fixed points and relaxation oscillations regimes are addressed. Some numerical examples are given and discussed in order to compare and to complete the asymptotic analysis in Sect. 4. Finally, the paper is concluded in Sect. 5.

\section{The system under study}

\subsection{The mechanical model}

The system considered here is shown in Fig. 1. It is composed of a primary system coupled to a NES in an ungrounded configuration. The primary system is defined by two 2-DOF Hulten systems with respective masses $m_{1}$ and $m_{2}$ which are linearly coupled by a spring of stiffness $k_{a}$, and a viscous damper of damping coefficient $c_{a}$. The two masses are arranged against three moving belts. It is assumed that the belts run at a constant speed, that the masses and the belts are always in contact and that the relative velocities between the masses and the belts are always positive so that the directions of the tangential fiction forces do not change. Hence, the tangential forces $F_{T}$ due to friction contacts are proportional to the normal forces $F_{N}$ as given by the Coulomb's law: $F_{T}=\gamma F_{N}$, where $\gamma$ is the friction coefficient. This 4-DOF linear model was introduced in [32] and used in [33] to illustrate different stability behaviors characterized by mode coupling having coalescence patterns. The number of DOF of the primary system corresponds to the minimal number of DOF which can be used to investigate the case of multi-instabilities.

The NES is simply modeled as a 1-DOF nonlinear oscillator characterized by a mass $m_{h}$, no linear stiffness, a cubic stiffness $k_{h}^{N L}$ and a damping coefficient $c_{h}$. 


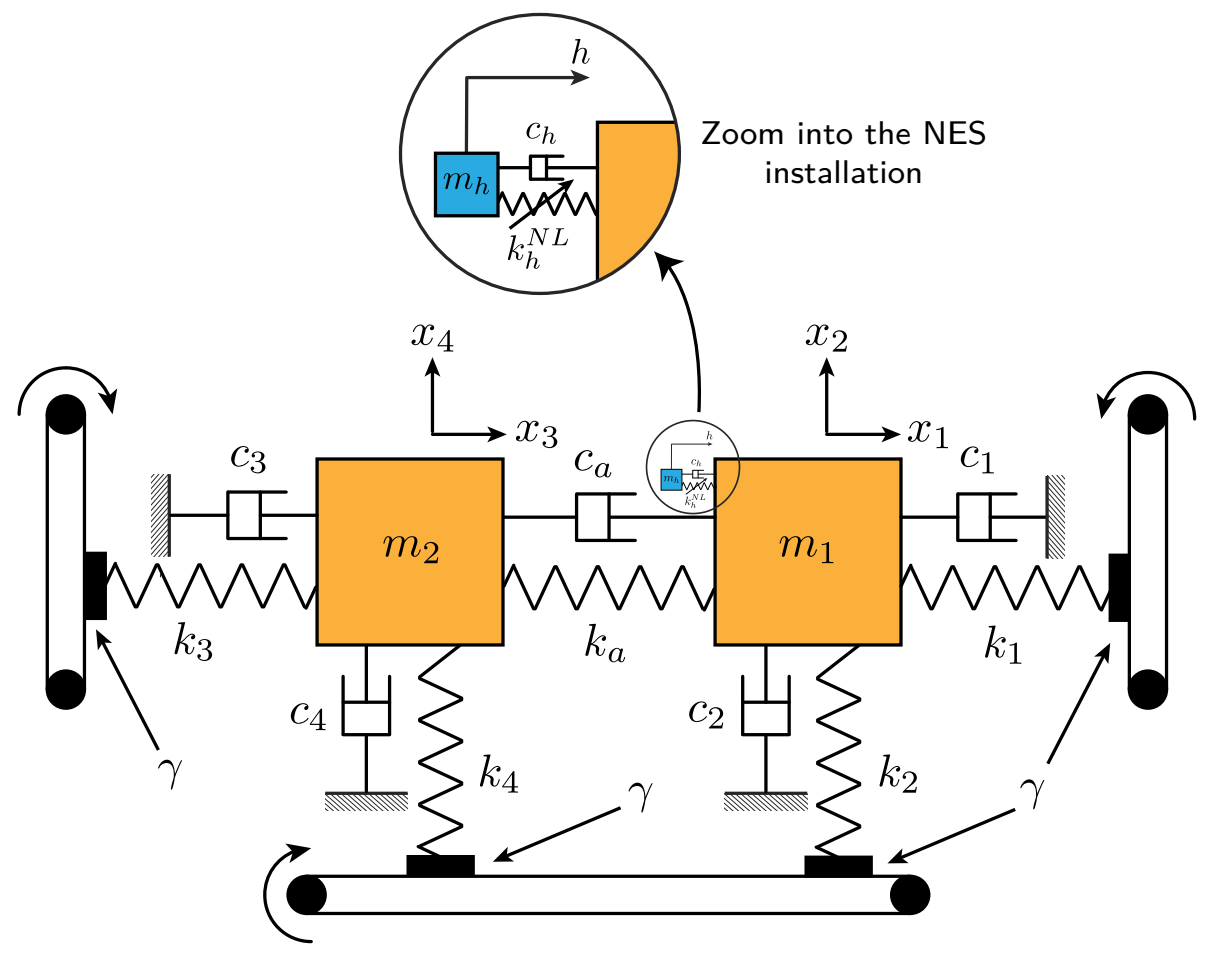

Figure 1: The phenomenological system under study.

The system is described by the following equations of motion

$$
\begin{array}{r}
m_{1} \frac{d^{2} \tilde{x}_{1}}{d \tilde{\tau}^{2}}+c_{1} \frac{d \tilde{x}_{1}}{d \tilde{\tau}}+c_{a}\left(\frac{d \tilde{x}_{1}}{d \tilde{\tau}}-\frac{d \tilde{x}_{3}}{d \tilde{\tau}}\right)+k_{1} \tilde{x}_{1}+ \\
k_{a}\left(\tilde{x}_{1}-\tilde{x}_{3}\right)+\gamma k_{2} \tilde{x}_{2}+m_{h} \frac{d^{2} \tilde{h}}{d \tilde{\tau}^{2}}=0 \\
m_{1} \frac{d^{2} \tilde{x}_{2}}{d \tilde{\tau}^{2}}+c_{2} \frac{d \tilde{x}_{2}}{d \tilde{\tau}}+k_{2} \tilde{x}_{2}-\gamma k_{1} \tilde{x}_{1}=0 \\
m_{2} \frac{d^{2} \tilde{x}_{3}}{d \tilde{\tau}^{2}}+c_{3} \frac{d \tilde{x}_{3}}{d \tilde{\tau}}+c_{a}\left(\frac{d \tilde{x}_{3}}{d \tilde{\tau}}-\frac{d \tilde{x}_{1}}{d \tilde{\tau}}\right)+k_{3} \tilde{x}_{3}+ \\
m_{2} \frac{d^{2} \tilde{x}_{4}}{d \tilde{\tau}^{2}}+c_{4} \frac{\left.d \tilde{x}_{3}-\tilde{x}_{1}\right)+\gamma k_{4} \tilde{x}_{4}=0}{d \tilde{\tau}}+k_{4} \tilde{x}_{4}-\gamma k_{3} \tilde{x}_{3}=0 \\
m_{h} \frac{d^{2} \tilde{h}}{d \tilde{\tau}^{2}}+c_{h}\left(\frac{d \tilde{h}}{d \tilde{\tau}}-\frac{d \tilde{x}_{1}}{d \tilde{\tau}}\right)+k_{h}^{N L}\left(\tilde{h}-\tilde{x}_{1}\right)^{3}=0
\end{array}
$$

where $\tilde{x}_{1}$ and $\tilde{x}_{2}$ (respectively $\tilde{x}_{3}$ and $\tilde{x}_{4}$ ) are the displacements of the primary mass $m_{1}$ (respectively $m_{2}$ ) and $\tilde{h}$ is the displacement of the NES. 
Introducing the following notations

$$
\begin{aligned}
\omega_{1} & =\sqrt{\frac{k_{1}}{m_{1}}}, \omega_{2}=\sqrt{\frac{k_{2}}{m_{1}}}, \omega_{3}=\sqrt{\frac{k_{3}}{m_{2}}}, \omega_{4}=\sqrt{\frac{k_{4}}{m_{2}}} \\
\eta_{i} & =\frac{c_{1}}{\omega_{1} m_{1}}, \eta_{2}=\frac{c_{2}}{\omega_{2} m_{1}}, \eta_{3}=\frac{c_{3}}{\omega_{3} m_{2}}, \eta_{4}=\frac{c_{4}}{\omega_{4} m_{2}} \\
\Omega_{i} & =\frac{\omega_{i}}{\omega_{1}} \text { for } i=2, \ldots, 4 \\
\sigma & =\frac{m_{2}}{m_{1}}, \Omega_{a}=\frac{k_{a}}{\omega_{1} m_{1}}, \eta_{a}=\frac{c_{a}}{\omega_{1} m_{1}}, \\
\tilde{\mu} & =\frac{c_{h}}{\omega_{1} m_{1}}, \alpha=\frac{k_{h}^{N L}}{\omega_{1}^{2} m_{1}}, \epsilon=\frac{m_{h}}{m_{1}}
\end{aligned}
$$

we can then rescale the variables $\tilde{x}_{i}$, for $i=1$ to 4 and $\tilde{h}$ as

$$
x_{i}=\frac{\tilde{x}_{i}}{\sqrt{\epsilon}} \text { for } i=1, \ldots, 4 \text { and } h=\frac{\tilde{h}}{\sqrt{\epsilon}},
$$

and change the time scale from $\tilde{\tau}$ to $t=\omega_{1} \tilde{\tau}$. Denoting as ". the derivative with respect to time $t$, the system of equations (1) takes the following final form

$$
\begin{aligned}
\ddot{x}_{1}+\left(\eta_{1}+\eta_{a}\right) \dot{x}_{1}-\eta_{a} \dot{x}_{3}+\left(1+\Omega_{a}^{2}\right) x_{1}+\gamma \Omega_{2}^{2} x_{2}-\Omega_{a}^{2} x_{3}+\epsilon \vec{h} & =0 \\
\ddot{x}_{2}+\eta_{2} \Omega_{2} \dot{x}_{2}-\gamma x_{1}+\Omega_{2}^{2} x_{2} & =0 \\
\ddot{x}_{3}-\frac{\eta_{a}}{\sigma} \dot{x}_{1}+\left(\eta_{3} \Omega_{3}+\frac{\eta_{a}}{\sigma}\right) \dot{x}_{3}-\frac{\Omega_{a}^{2}}{\sigma} x_{1}+\left(\Omega_{3}^{2}+\frac{\Omega_{a}^{2}}{\sigma}\right) x_{3}+\gamma \Omega_{4}^{2} x_{4} & =0 \\
\ddot{x}_{4}+\eta_{4} \Omega_{4} \dot{x}_{4}-\gamma \Omega_{3}^{2} x_{3}+\Omega_{4}^{2} x_{4} & =0 \\
\ddot{h}+\mu\left(\dot{h}-\dot{x}_{1}\right)+\alpha\left(h-x_{1}\right)^{3} & =0
\end{aligned}
$$

where $\tilde{\mu}$ has been rescaled as $\tilde{\mu}=\epsilon \mu$.

From now on we assume that $\epsilon$ is small. As it also implies that $\tilde{\mu}$ is small, this assumption is satisfied if the mass ratio and the damping coefficient of the NES are small.

\subsection{The reduced model}

To simplify the following calculations, it is convenient to introduce new coordinates in (3) as

$$
u_{1}=x_{1}+\epsilon h, \quad u_{i}=x_{i} \quad \text { for } \quad i=2, \ldots, 4 \quad \text { and } \quad v=x_{1}-h
$$

leading, after a first-order Taylor expansion around $\epsilon=0$, to the following equations

$$
\begin{array}{r}
\ddot{u}_{1}+\left(\eta_{1}+\eta_{a}\right) \dot{u}_{1}-\eta_{a} \dot{u}_{3}+\left(1+\Omega_{a}^{2}\right) u_{1}+\gamma \Omega_{2}^{2} u_{2}-\Omega_{a}^{2} u_{3}+ \\
\epsilon\left[-\left(\eta_{1}+\eta_{a}\right) \dot{u}_{1}+\left(\eta_{1}+\eta_{a}\right) \dot{v}-\left(1+\Omega_{a}^{2}\right) u_{1}+\left(1+\Omega_{a}^{2}\right) v\right]=0 \\
\ddot{u}_{2}+\eta_{2} \Omega_{2} \dot{u}_{2}-\gamma u_{1}+\Omega_{2}^{2} u_{2}+\epsilon \gamma\left(u_{1}-v\right)=0 \\
\ddot{u}_{3}-\frac{\eta_{a}}{\sigma} \dot{u}_{1}+\left(\eta_{3} \Omega_{3}+\frac{\eta_{a}}{\sigma}\right) \dot{u}_{3}-\frac{\Omega_{a}^{2}}{\sigma} u_{1}+\gamma \Omega_{4}^{2} u_{4}+\left(\Omega_{3}^{2}+\frac{\Omega_{a}^{2}}{\sigma}\right) u_{3}+ \\
\frac{\epsilon}{\sigma}\left[\eta_{a} \dot{u}_{1}-\eta_{a} \dot{v}+\Omega_{a}^{2} u_{1}-\Omega_{a}^{2} v\right]=0 \\
\ddot{u}_{4}+\eta_{4} \Omega_{4} \dot{u}_{4}-\gamma \Omega_{3}^{2} u_{3}+\Omega_{4}^{2} u_{4}=0 \\
\ddot{v}+\left(\eta_{1}+\eta_{a}\right) \dot{u}_{1}-\eta_{a} \dot{u}_{3}+\mu \dot{v}+\left(1+\Omega_{a}^{2}\right) u_{1}+\gamma \Omega_{2}^{2} u_{2}-\Omega_{a}^{2} u_{3}+\alpha v^{3}+ \\
\epsilon\left[-\left(\eta_{1}+\eta_{a}\right) \dot{u}_{1}+\left(\eta_{1}+\eta_{a}\right) \dot{v}+\mu \dot{v}-\left(1+\Omega_{a}^{2}\right) u_{1}+\left(1+\Omega_{a}^{2}\right) v+\alpha v^{3}\right]=0 .
\end{array}
$$


Then, to capture the essential features of the double instability of the primary system, a biorthogonal transformation is applied to the variables characterizing the primary system after writing Eqs. (5a) to (5d) in state-space form:

$$
\dot{\mathbf{y}}=\mathbf{A y}+\epsilon\left(\mathbf{D}_{1} \mathbf{y}+\mathbf{D}_{2} v+\mathbf{D}_{3} \dot{v}\right)
$$

where $\mathbf{y}=\left(u_{1}, \cdots, u_{4}, \dot{u}_{1}, \cdots, \dot{u}_{4}\right)^{T}$. The matrices $\mathbf{A}$ and $\mathbf{D}_{1}$ and the vectors $\mathbf{D}_{2}$ and $\mathbf{D}_{3}$ are not explicitly given but can be easily obtained from (5a-5d). The non symmetric matrix $\mathbf{A}$ characterizes the dynamics of the primary system.

Let us consider the right and left eigenvector matrices

$$
\mathbf{R}=\left[\begin{array}{lllll}
\mathbf{r}_{\mathbf{1}} & \mathbf{r}_{\mathbf{1}}^{*} & \cdots & \mathbf{r}_{\mathbf{4}} & \mathbf{r}_{4}^{*}
\end{array}\right] \text { and } \mathbf{L}=\left[\begin{array}{lllll}
\mathbf{l}_{\mathbf{1}} & \mathbf{l}_{\mathbf{1}}^{*} & \cdots & \mathbf{l}_{\mathbf{2}} & \mathbf{l}_{\mathbf{4}}^{*}
\end{array}\right]
$$

corresponding to the following right and left eigenvalue problems with respect to the matrix $\mathbf{A}$

$$
\mathbf{A R}=\mathbf{R} \boldsymbol{\Lambda} \text { and } \quad \mathbf{A}^{T} \mathbf{L}=\mathbf{L} \boldsymbol{\Lambda}
$$

where $\boldsymbol{\Lambda}=\operatorname{diag}\left(\lambda_{1}, \lambda_{1}^{*}, \cdots, \lambda_{4}, \lambda_{4}^{*}\right)$ denotes the diagonal matrix of the eigenvalues. The superscript $(.)^{*}$ denotes the complex conjugate.

From now on we assume that the primary system is unstable with two pairs of conjugate eigenvalues having small positive real parts, we arrange the eigenvalues so that $\lambda_{1}^{R}>0$ and $\lambda_{2}^{R}>0$ where $\lambda_{i}=\lambda_{i}^{R}+j \lambda_{i}^{I}$ for $i=1,2$ and we assume that

$$
\lambda_{1}^{R}=\epsilon \rho_{1} \quad \text { and } \quad \lambda_{2}^{R}=\epsilon \rho_{2}
$$

with $\rho_{1}$ and $\rho_{2}$ of the order of $O(1)$. The two other pairs of eigenvalues have negative real parts.

We introduce the biorthogonal transformation

$$
\mathbf{y}=\mathbf{R q} \text { with } \mathbf{q}=\mathbf{L}^{T} \mathbf{y}
$$

where the vector $\mathbf{q}=\left(q_{1}, q_{1}^{*}, \cdots, q_{4}, q_{4}^{*}\right)^{T}$ is constituted of four pairs of complex conjugates. The matrices $\mathbf{R}$ and $\mathbf{L}$ have been chosen such that

$$
\mathbf{L}^{T} \mathbf{R}=\mathbf{I} \quad \text { and } \quad \mathbf{L}^{\mathbf{T}} \mathbf{A} \mathbf{R}=\boldsymbol{\Lambda} .
$$

where $\mathbf{I}$ denotes the identity matrix.

We can deduce that Eqs. (5) take the following formal form with respect to the new variables q:

$$
\begin{aligned}
\dot{\mathbf{q}}-\boldsymbol{\Lambda} \mathbf{q}-\epsilon \mathbf{f}(\mathbf{q}, v, \dot{v}) & =0 \\
\ddot{v}+g(v, \dot{v}, \mathbf{q}, \epsilon) & =0
\end{aligned}
$$

where $\mathbf{f}=\mathbf{L}^{T}\left(\mathbf{D}_{1} \mathbf{R q}+\mathbf{D}_{2} v+\mathbf{D}_{3} \dot{v}\right)$. Again, the function $g$ is not explicitly given but can be easily obtained from $(5 \mathrm{e})$.

Neglecting the coupling term $\epsilon \mathbf{f}(\mathbf{q}, v, \dot{v})$, Eq. (12a) reduces to $\dot{\mathbf{q}}-\mathbf{\Lambda} \mathbf{q}=0$ and the variables $q_{3}, q_{3}^{*}, q_{4}$ and $q_{4}^{*}$, related to the stable modes, vanish (i.e. tend to zero). When the coupling term $\epsilon \mathbf{f}(\mathbf{q}, v, \dot{v})$, which is of the order of magnitude of $\epsilon$, is taken into account, the variables $q_{3}, q_{3}^{*}, q_{4}$ and $q_{4}^{*}$ do not vanish but their contributions remain minimal and can be neglected $[9,22]$. Consequently, all the terms related to $q_{3}, q_{3}^{*}, q_{4}$ and $q_{4}^{*}$ will be omitted in the following developments.

Eq. (12) may therefore be simplified, retaining only the components $q_{1}, q_{1}^{*}, q_{2}$ and $q_{2}^{*}$ associated with 
the unstable eigenvalues. The resulting equations take the (reduced) form

$$
\begin{array}{r}
\dot{q}_{1}-j \lambda_{1}^{I} q_{1}-\epsilon \rho_{1} q_{1}- \\
\epsilon \mathbf{L}_{1 .}^{T}\left[\mathbf{D}_{1}\left(\mathbf{R}_{1 .} q_{1}+\mathbf{R}_{2 .} q_{1}^{*}+\mathbf{R}_{3 .} q_{2}+\mathbf{R}_{4 .} q_{2}^{*}\right)+\mathbf{D}_{2} v+\mathbf{D}_{3} \dot{v}\right]=0 \\
\dot{q}_{2}-j \lambda_{2}^{I} q_{2}-\epsilon \rho_{2} q_{2}- \\
\epsilon \mathbf{L}_{3 .}^{T}\left[\mathbf{D}_{1}\left(\mathbf{R}_{1 .} q_{1}+\mathbf{R}_{2 .} q_{1}^{*}+\mathbf{R}_{3 .} q_{2}+\mathbf{R}_{4 .} q_{2}^{*}\right)+\mathbf{D}_{2} v+\mathbf{D}_{3} \dot{v}\right]=0 \\
\ddot{v}+\mu \dot{v}+\alpha v^{3}+ \\
\left(\eta_{1}+\eta_{a}\right)\left(R_{51} q_{1}+R_{51}^{*} q_{1}^{*}+R_{53} q_{2}+R_{53}^{*} q_{2}^{*}\right)- \\
\eta_{a}\left(R_{71} q_{1}+R_{71}^{*} q_{1}^{*}+R_{73} q_{2}+R_{73}^{*} q_{2}^{*}\right)+ \\
\left(1+\Omega_{a}^{2}\right)\left(R_{11} q_{1}+R_{11}^{*} q_{1}^{*}+R_{13} q_{2}+R_{13}^{*} q_{2}^{*}\right)+ \\
\gamma \Omega_{2}^{2}\left(R_{21} q_{1}+R_{21}^{*} q_{1}^{*}+R_{23} q_{2}+R_{23}^{*} q_{2}^{*}\right)- \\
\Omega_{a}^{2}\left(R_{31} q_{1}+R_{31}^{*} q_{1}^{*}+R_{33} q_{2}+R_{33}^{*} q_{2}^{*}\right)+ \\
\epsilon\left[\left(\eta_{1}+\eta_{a}+\mu\right) \dot{v}+\left(1+\Omega_{a}^{2}\right) v+\alpha v^{3}-\right. \\
\left(\eta_{1}+\eta_{a}\right)\left(R_{51} q_{1}+R_{51}^{*} q_{1}^{*}+R_{53} q_{2}+R_{53}^{*} q_{2}^{*}\right)- \\
\left.\left(1+\Omega_{a}^{2}\right)\left(R_{11} q_{1}+R_{11}^{*} q_{1}^{*}+R_{13} q_{2}+R_{13}^{*} q_{2}^{*}\right)\right]=0
\end{array}
$$

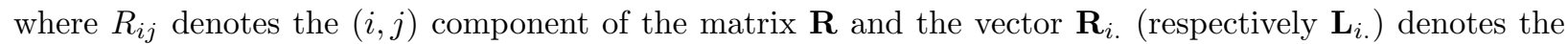
$i^{\text {th }}$ column of the matrix $\mathbf{R}^{T}$ (respectively $\mathbf{L}^{T}$ ). Eq. (13) involves two complex variables $q_{1}$ and $q_{2}$ and one real variable $v$.

\section{Asymptotic analysis}

The objective of this section is to characterize analytically the response regimes of the reduced model (13) in the simultaneous vicinity of the unstable natural frequencies $\lambda_{1}^{I}$ and $\lambda_{2}^{I}$. The proposed method which uses $\epsilon$ as a small parameter includes two steps. The first step (Sect. 3.1) combines complexification and averaging methods to reduce the dynamics of the nonlinear system (13) by removing the oscillations with respect to the frequencies $\lambda_{1}^{I}$ and $\lambda_{2}^{I}$. The resulting dynamical system appears as a slow-fast system where the fast dynamics corresponds to the current time $t$ whereas the slow dynamics is related to the time $\epsilon t$. Note that the dynamics with respect to the frequencies $\lambda_{1}^{I}$ and $\lambda_{2}^{I}$ defines a third time scale denoted as the super fast dynamics. The reduced system can be analyzed using the geometric singular perturbation theory which is the objective of the second step (Sects. 3.2 to 3.5). That step begins with the definition of the critical manifold (Sect. 3.2), which defines the space where the slow flow takes place. The associated stability properties are then considered in Sect. 3.3. It continues with the characterization of the periodic or quasi-periodic regimes of the mechanical system (5) as stable fixed points of the slow flow (19) (Sect. 3.4). Finally, in Sect. 3.5, it is shown that the specific shape of the critical manifold enables the existence of more complicate steady-state responses named "relaxations oscillations", which exhibit strong modulated amplitudes on both frequency components.

\subsection{The complexification-averaging method and the slow-fast system}

Our objective is to investigate the solution in the vicinity of two simultaneous natural frequencies $\lambda_{1}^{I}$ and $\lambda_{2}^{I}$ named 1:1-1:1 resonances. We assume that $\lambda_{1}^{I}$ and $\lambda_{2}^{I}$ are incommensurable.

The complexification-averaging method [34] is used to make the slow dynamics of the system emerge from fast oscillating responses.

The complex modal variable $q_{1}$ (respectively $q_{2}$ ) may be written as a fast component with respect to the natural frequency $\lambda_{1}^{I}$ (respectively $\lambda_{2}^{I}$ ), and modulated by a slowly varying amplitude $\phi_{1}$ (respectively $\phi_{2}$ ), 
that is

$$
\begin{array}{lll}
q_{1}=\phi_{1} e^{j \lambda_{1}^{I} t} & \text { and } & \dot{q}_{1}=\dot{\phi}_{1} e^{j \lambda_{1}^{I} t}+j \phi_{1} \lambda_{1}^{I} e^{j \lambda_{1}^{I} t} \\
q_{2}=\phi_{2} e^{j \lambda_{2}^{I} t} & \text { and } & \dot{q}_{2}=\dot{\phi}_{2} e^{j \lambda_{2}^{I} t}+j \phi_{2} \lambda_{2}^{I} e^{j \lambda_{2}^{I} t} .
\end{array}
$$

As for the real variable $v$, new variables $v_{1}$ and $v_{2}$ are introduced as

$$
v=v_{1}+v_{2}
$$

to capture frequency components with respect to $\lambda_{1}^{I}$ and $\lambda_{2}^{I}$ (fast components) respectively. The slowly changing complex amplitudes $\xi_{1}$ and $\xi_{2}$ are then respectively defined as

$$
\begin{aligned}
& \xi_{1} e^{j \lambda_{1}^{I} t}=\dot{v}_{1}+j \lambda_{1}^{I} v_{1} \\
& \xi_{2} e^{j \lambda_{2}^{I} t}=\dot{v}_{2}+j \lambda_{2}^{I} v_{2} .
\end{aligned}
$$

Inserting Eqs. (14), (15) and (16) into Eqs. (13) and averaging with respect to the frequencies $\lambda_{1}^{I}$ and $\lambda_{2}^{I}$ separately yields the following system of complex slow modulation with respect to $\left(\phi_{1}, \phi_{2}, \xi_{1}, \xi_{2}\right)$ :

$$
\begin{aligned}
\dot{\phi}_{1} & =\epsilon F_{1}\left(\phi_{1}, \xi_{1}\right) \\
\dot{\phi}_{2} & =\epsilon F_{2}\left(\phi_{2}, \xi_{2}\right) \\
\dot{\xi}_{1} & =G_{1}\left(\phi_{1}, \xi_{1}, \xi_{2}, \epsilon\right) \\
\dot{\xi}_{2} & =G_{2}\left(\phi_{2}, \xi_{2}, \xi_{1}, \epsilon\right)
\end{aligned}
$$

where the complex functions $F_{1}, F_{2}, G_{1}$ and $G_{2}$ are not explicitly given. Eqs. (17) characterize the dynamics of the slow complex amplitudes $\phi_{1}, \phi_{2}, \xi_{1}$ and $\xi_{2}$ of the physical variables $q_{1}, q_{2}, v_{1}$ and $v_{2}$ respectively.

By expanding Eqs. (17) using the following polar coordinates

$$
\begin{aligned}
\phi_{1} & =s_{1} e^{j \delta_{1}} \\
\phi_{2} & =s_{2} e^{j \delta_{2}} \\
\xi_{1} & =r_{1} e^{j \theta_{1}} \\
\xi_{2} & =r_{2} e^{j \theta_{2}},
\end{aligned}
$$

new equations of motion in terms of amplitudes $s_{1}, s_{2}, r_{1}$ and $r_{2}$ and phase differences $\Delta_{1}=\delta_{1}-\theta_{1}$ and $\Delta_{2}=\delta_{2}-\theta_{2}$ can be obtained as

$$
\begin{aligned}
& \dot{\mathbf{s}}=\epsilon \mathbf{f}(\mathbf{s}, \mathbf{t}) \\
& \dot{\mathbf{t}}=\mathbf{g}(\mathbf{s}, \mathbf{t}, \epsilon)=\mathbf{g}_{0}(\mathbf{s}, \mathbf{t})+\epsilon \mathbf{g}_{1}(\mathbf{s}, \mathbf{t})
\end{aligned}
$$

where $\mathbf{s}=\left(s_{1}, s_{2}\right)^{T}$ and $\mathbf{t}=\left(r_{1}, r_{2}, \Delta_{1}, \Delta_{2}\right)^{T}$. The functions $\mathbf{f}=\left(f_{1}, f_{2}\right)^{T}, \mathbf{g}_{0}=\left(g_{01}, g_{02}, g_{03}, g_{04}\right)^{T}$ and $\mathbf{g}_{1}=\left(g_{11}, g_{21}, g_{31}, g_{41}\right)^{T}$ take the following forms

$$
\begin{aligned}
f_{1}\left(s_{1}, r_{1}, \Delta_{1}\right) & =a_{1} s_{1}+r_{1}\left(a_{2} \cos \Delta_{1}+a_{3} \sin \Delta_{1}\right) \\
f_{2}\left(s_{2}, r_{2}, \Delta_{2}\right) & =a_{4} s_{2}+r_{2}\left(a_{5} \cos \Delta_{2}+a_{6} \sin \Delta_{2}\right) \\
g_{01}\left(s_{1}, r_{1}, \Delta_{1}\right) & =H_{01}\left(r_{1}\right)+s_{1}\left(a_{7} \cos \Delta_{1}+a_{8} \sin \Delta_{1}\right) \\
g_{02}\left(s_{2}, r_{2}, \Delta_{2}\right) & =H_{02}\left(r_{2}\right)+s_{2}\left(a_{9} \cos \Delta_{2}+a_{10} \sin \Delta_{2}\right) \\
g_{03}\left(s_{1}, r_{1}, \Delta_{1}, r_{2}\right) & =\frac{1}{r_{1}}\left(H_{03}\left(r_{1}, r_{2}\right)+s_{1}\left(a_{8} \cos \Delta_{1}-a_{7} \sin \Delta_{1}\right)\right) \\
g_{04}\left(s_{2}, r_{2}, \Delta_{2}, r_{1}\right) & =\frac{1}{r_{2}}\left(H_{04}\left(r_{1}, r_{2}\right)+s_{2}\left(a_{10} \cos \Delta_{2}-a_{9} \sin \Delta_{2}\right)\right),
\end{aligned}
$$


with

$$
\begin{aligned}
H_{01}\left(r_{1}\right) & =-\frac{\mu}{2} r_{1}, \\
H_{02}\left(r_{2}\right) & =-\frac{\mu}{2} r_{2}, \\
H_{03}\left(r_{1}, r_{2}\right) & =r_{1}\left(\frac{\lambda_{1}^{I}}{2}-\frac{3 \alpha}{8 \lambda_{1}^{I^{3}}} r_{1}^{2}-\frac{3 \alpha}{4 \lambda_{1}^{I} \lambda_{2}^{I^{2}}} r_{2}^{2}\right), \\
H_{04}\left(r_{1}, r_{2}\right) & =r_{2}\left(\frac{\lambda_{2}^{I}}{2}-\frac{3 \alpha}{4 \lambda_{1}^{I^{2}} \lambda_{2}^{I}} r_{1}^{2}-\frac{3 \alpha}{8 \lambda_{2}^{I^{3}}} r_{2}^{2}\right) .
\end{aligned}
$$

The coefficients $a_{i}$ for $i=1, \ldots, 10$, are reported in Appendix $\mathrm{A}$ and the other functions are not given explicitly because they are not used in the following.

In the framework of the Geometric Singular Perturbation Theory, Eqs. (19) define a (4,2)-fast-slow system where $\mathbf{t}=\left(r_{1}, r_{2}, \Delta_{1}, \Delta_{2}\right)^{T}$ denote the fast variables and $\mathbf{s}=\left(s_{1}, s_{2}\right)^{T}$ the slow ones. The slow variables are related to the modal components $q_{1}$ and $q_{2}$ whereas the fast variables are associated with the frequency components $v_{1}$ and $v_{2}$ of the NES variable $v$.

The system (19) corresponds to the fast time scale whereas for the slow time scale $\tau=\epsilon t$, the system reads

$$
\begin{aligned}
\mathbf{s}^{\prime} & =\mathbf{f}(\mathbf{s}, \mathbf{t}) \\
\epsilon \mathbf{t}^{\prime} & =\mathbf{g}(\mathbf{s}, \mathbf{t}, \epsilon)=\mathbf{g}_{0}(\mathbf{s}, \mathbf{t})+\epsilon \mathbf{g}_{1}(\mathbf{s}, \mathbf{t})
\end{aligned}
$$

where $(.)^{\prime}$ denotes the derivative with respect to $\tau$. The slow and the fast subsystems are defined by considering $\epsilon=0$, which yields for the slow subsystem

$$
\begin{aligned}
\mathbf{s}^{\prime} & =\mathbf{f}(\mathbf{s}, \mathbf{t}) \\
\mathbf{0} & =\mathrm{g}_{0}(\mathbf{s}, \mathbf{t}),
\end{aligned}
$$

which is a differential-algebraic equation, and for the fast subsystem

$$
\begin{aligned}
\dot{\mathrm{s}} & =\mathbf{0} \\
\dot{\mathrm{t}} & =\mathrm{g}_{0}(\mathrm{~s}, \mathrm{t}) .
\end{aligned}
$$

The slow and the fast subsystems will be complementary in the following analysis. Periodic or quasiperiodic steady-state regimes of the mechanical system (13) correspond to fixed points of the slow flow which are approximated by those of the slow subsystem. Indeed, if a fixed point of the slow flow with non-zero amplitude coordinates (i.e. $s_{1}, s_{2}, r_{1}$ and $r_{2}$ ) is reached, the resulting steady-sate regime of the system (13) is approximated by a quasi-periodic regime containing the two harmonic components at the frequencies $\lambda_{1}^{I}$ and $\lambda_{2}^{I}$ respectively. If a fixed point with $s_{1}=r_{1}=0$ (respectively $s_{2}=r_{2}=0$ ) is reached, the resulting steady-sate regime is approximated by a periodic regime containing only one harmonic component at the frequency $\lambda_{2}^{I}$ (respectively $\lambda_{1}^{I}$ ).

More complicate (amplitude and phase modulated) steady-state regimes of the mechanical system (13) will be explained, inter alia, by the existence of relaxation oscillations of the slow flow (see Sect. 3.5), which are described combining slow and fast periods predicted by (31) and (32) respectively. In the case of only one unstable mode, those regimes are referred to as Strongly Modulated Responses (SMR) [35]. This term is kept in this work but it covers more situations which are described in Sect. 3.5. Although SMR are also quasi-periodic regimes, we have chosen to reserve the "quasi-periodic" term for the situations evoked above in which a fixed point of the slow flow is reached.

\subsection{The critical manifold}

The critical manifold is given as

$$
C_{0}:=\left\{(\mathbf{s}, \mathbf{t}) \in \mathbb{R}^{2} \times \mathbb{R}^{4} \mid \mathbf{g}_{0}(\mathbf{s}, \mathbf{t})=0\right\} .
$$


It defines the domain where the slow flow takes place (see (31b)) and consists in the equilibrium of the fast flow (see $(32 \mathrm{~b})$ ). It plays a crucial role to characterize the response regimes under asymptotic assumptions.

The equations defining the critical manifold (33) can be simplified solving $g_{01}=0$ and $g_{03}=0$ (respectively $g_{02}=0$ and $\left.g_{04}=0\right)$ with respect to $\cos \Delta_{1}$ and $\sin \Delta_{1}\left(\right.$ respectively $\cos \Delta_{2}$ and $\left.\sin \Delta_{2}\right)$, which gives

$$
\cos \Delta_{1}=-\frac{a_{8} H_{03}\left(r_{1}, r_{2}\right)+a_{7} H_{01}\left(r_{1}\right)}{s_{1}\left(a_{7}^{2}+a_{8}^{2}\right)} \quad \text { and } \quad \sin \Delta_{1}=-\frac{a_{8} H_{01}\left(r_{1}\right)-a_{7} H_{03}\left(r_{1}, r_{2}\right)}{s_{1}\left(a_{7}^{2}+a_{8}^{2}\right)}
$$

(respectively

$$
\left.\cos \Delta_{2}=-\frac{a_{10} H_{04}\left(r_{1}, r_{2}\right)+a_{9} H_{02}\left(r_{1}\right)}{s_{2}\left(a_{9}^{2}+a_{10}^{2}\right)} \quad \text { and } \quad \sin \Delta_{2}=-\frac{a_{10} H_{02}\left(r_{1}\right)-a_{9} H_{04}\left(r_{1}, r_{2}\right)}{s_{2}\left(a_{9}^{2}+a_{10}^{2}\right)}\right) .
$$

Finally, compatibility equations relating $s_{1}$ and $s_{2}$ with $r_{1}$ and $r_{2}$ can be deduced from (34) and (35) as

$$
\begin{aligned}
& s_{1}^{2}=\frac{H_{1}\left(r_{1}, r_{2}\right)}{a_{7}^{2}+a_{8}^{2}} \\
& s_{2}^{2}=\frac{H_{2}\left(r_{1}, r_{2}\right)}{a_{9}^{2}+a_{10}^{2}}
\end{aligned}
$$

where

$$
\begin{aligned}
& H_{1}\left(r_{1}, r_{2}\right)=H_{01}^{2}\left(r_{1}\right)+H_{03}^{2}\left(r_{1}, r_{2}\right) \\
& H_{2}\left(r_{1}, r_{2}\right)=H_{02}^{2}\left(r_{2}\right)+H_{04}^{2}\left(r_{1}, r_{2}\right) .
\end{aligned}
$$

The critical manifold $C_{0}$ relates the variables $s_{1}, s_{2}, \Delta_{1}$ and $\Delta_{2}$ to $r_{1}$ and $r_{2}$ (see Eqs. (34) to (37)) and appears as a two-dimensional parametric surface with respect to the two fast variables $r_{1}$ and $r_{2}$ which evolve in the six-dimensional variable space. It is hence possible to plot $C_{0}$ as two surfaces by projecting it on the three-dimensional spaces $\left(r_{1}, r_{2}, s_{1}\right)$ and $\left(r_{1}, r_{2}, s_{2}\right)$ using Eq. (37a) and Eq. (37b) respectively. Figure 2 shows the corresponding two surfaces for given nominal parameter values of the considered system. The surface in the three-dimensional space $\left(r_{1}, r_{2}, s_{1}\right)$ (see Fig. $2(\mathrm{a})$ ) depicts $C_{0}$ with respect to the unstable mode $\lambda_{1}$. Note that, as observed when only one mode is unstable [20], $C_{0}$ restricted to $r_{2}=0$ is $S$-shaped. Similar comments can be made considering the surface in the three-dimensional space $\left(r_{1}, r_{2}, s_{2}\right)$ related to the unstable mode $\lambda_{2}$. The presence of an $S$-shaped critical manifold will be used to show that SMR steady-state responses of the mechanical system can exist (as in the case of only one unstable mode, see for example [20]).

Finally, the slow dynamics described by the slow subsystem can be only written in the $\left(r_{1}, r_{2}\right)$-plane, as performed hereafter in Sect. 3.4.

\subsection{Stability of critical manifold}

The points constituting $C_{0}$ are equilibrium point of the fast subsystem (32) and the stability properties can be analyzed from the Jacobian matrix of $\mathbf{g}_{0}(\mathbf{s}, \mathbf{t})$ with respect to $\mathbf{t}$, denoted as $D_{\mathbf{t}} \mathbf{g}_{0}(\mathbf{s}, \mathbf{t})$, and evaluated at each point of $C_{0}$ as

$$
D_{\mathbf{t}} \mathbf{g}_{0}(\mathbf{s}, \mathbf{t})=\left(\begin{array}{cccc}
\frac{d H_{01}}{d r_{1}}\left(r_{1}\right) & -H_{03}\left(r_{1}, r_{2}\right) & 0 & 0 \\
0 & 0 & \frac{d H_{02}}{d r_{2}}\left(r_{2}\right) & -H_{04}\left(r_{1}, r_{2}\right) \\
\frac{1}{r_{1}} \frac{\partial H_{03}}{\partial r_{1}}\left(r_{1}, r_{2}\right) & \frac{H_{01}\left(r_{1}\right)}{r_{1}} & \frac{1}{r_{1}} \frac{\partial H_{03}}{\partial r_{2}}\left(r_{1}, r_{2}\right) & 0 \\
\frac{1}{r_{2}} \frac{\partial H_{04}}{\partial r_{1}}\left(r_{1}, r_{2}\right) & 0 & \frac{1}{r_{2}} \frac{\partial H_{04}}{\partial r_{2}}\left(r_{1}, r_{2}\right) & \frac{H_{02}}{r_{2}}\left(r_{2}\right)
\end{array}\right) .
$$

The critical manifold $C_{0}$ contains both regular points defined as $\operatorname{det}\left(D_{\mathbf{t}} \mathbf{g}_{0}(\mathbf{s}, \mathbf{t})\right) \neq 0$ and singular points characterized by $\operatorname{det}\left(D_{\mathbf{t}} \mathbf{g}_{0}(\mathbf{s}, \mathbf{t})\right)=0$. Depending on the eigenvalues of $D_{\mathbf{t}} \mathbf{g}_{0}(\mathbf{s}, \mathbf{t})$, the set of regular points 


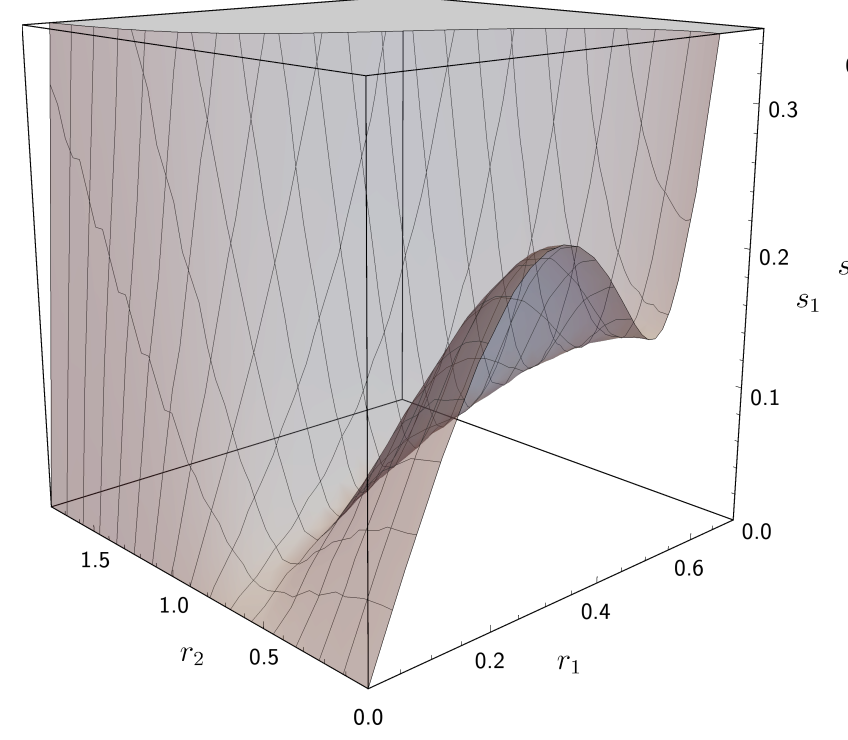

(a)

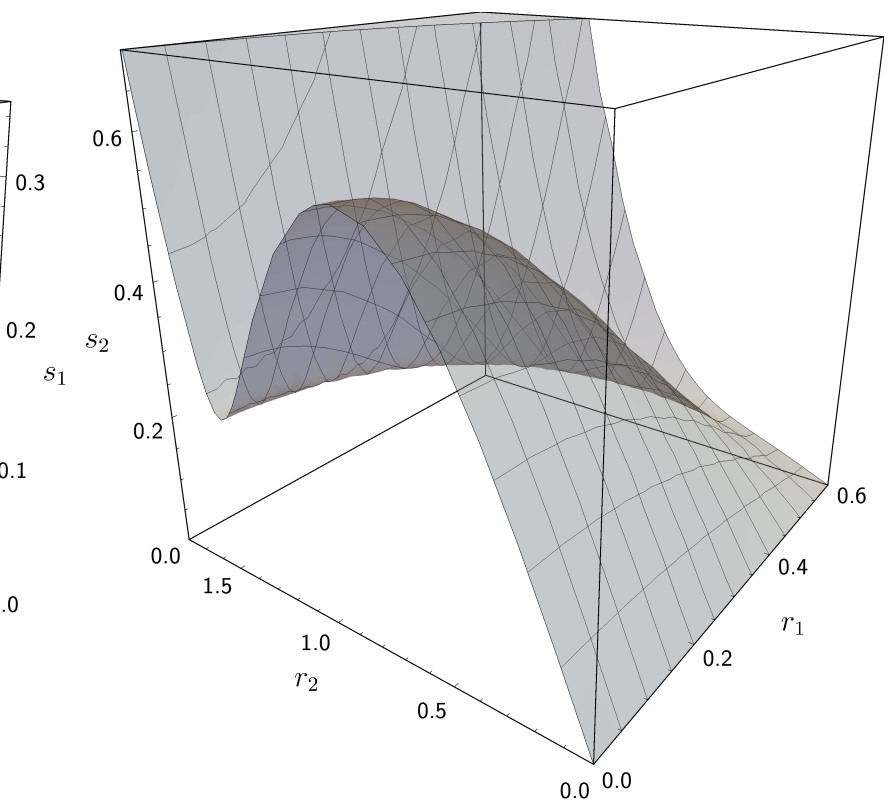

(b)

Figure 2: The critical manifold in (a) the $\left(r_{1}, r_{2}, s_{1}\right)$-space (function (36a)) and (b) in the $\left(r_{1}, r_{2}, s_{2}\right)$-space (function (36b)). The set of parameters used are given in Sect. 4.3 , Eq. (58).

can be split into several parts (see definitions in Chap. 3 of [36]), including normally hyperbolic attracting parts (all the eigenvalues have strictly negative real parts), normally hyperbolic repelling parts (all the eigenvalues have strictly positive real parts), normally hyperbolic saddle type parts (neither attracting nor repelling) and non normally hyperbolic parts (at least one eigenvalue has a zero real part). Finally, the singular points for which the rank of $D_{\mathbf{t}} \mathbf{g}_{0}(\mathbf{s}, \mathbf{t})$ is equal to $3(=4-1)$, (the simplest possible rank deficiency) are denoted as fold points.

In general normally hyperbolic parts of $C_{0}$ constitute surfaces in the $\left(r_{1}, r_{2}\right)$-plane whereas points that are not normally hyperbolic (i.e. regular non normally hyperbolic points and singular points) are not isolated but form a one-dimensional curve inside $C_{0}$.

Fig. 3 shows, for the same nominal parameter values of the considered system as above, the different parts of $C_{0}$ in the $\left(r_{1}, r_{2}\right)$-plane as

$$
C_{0}=C_{0, r}^{a} \cup\left(F^{1} \cup C_{0, r}^{s t, 1}\right) \cup\left(F^{2} \cup C_{0, r}^{s t, 2}\right) \cup\left(F^{3} \cup C_{0, r}^{s t, 3}\right) \cup\left(N_{r} \cup C_{0, r}^{s t, 4}\right)
$$

where $C_{0, r}^{a}$ (Fig. 3 - white background) is a (regular) normally hyperbolic attracting part and $C_{0, r}^{s t, 1}$ (respectively $C_{0, r}^{s t, 2}$ and $C_{0, r}^{s t, 3}$ ) (Fig. 3 - salmon background) is a (regular) normally hyperbolic saddle type part that meets $C_{0, r}^{a}$ at the fold curve $F^{1}$ (respectively $F^{2}$ and $F^{3}$ ) (Fig. 3 - dashed and continuous red lines). $C_{0, r}^{s t, 4}$ (Fig. 3 - green background) is a normally hyperbolic saddle type part that meets $C_{0, r}^{a}$ at a (regular) non normally hyperbolic curve $N_{r}$ (Fig. 3 - green line).

\subsection{Fixed points of the slow-flow in $C_{0}$}

As mentioned previously, the fixed points of the slow flow (19) characterize periodic or quasi-periodic regimes of the mechanical system (5). Since $0<\epsilon \ll 1$, these fixed points can be approximated by those of the slow subsystem (31) which are computed in this section.

The critical manifold $C_{0}$ relates the variables $s_{1}, s_{2}, \Delta_{1}$ and $\Delta_{2}$ to the variable $r_{1}$ and $r_{2}$. Hence, substituting Eq. (36) into (31), the dynamics of the slow subsystem can be considered only with respect to 

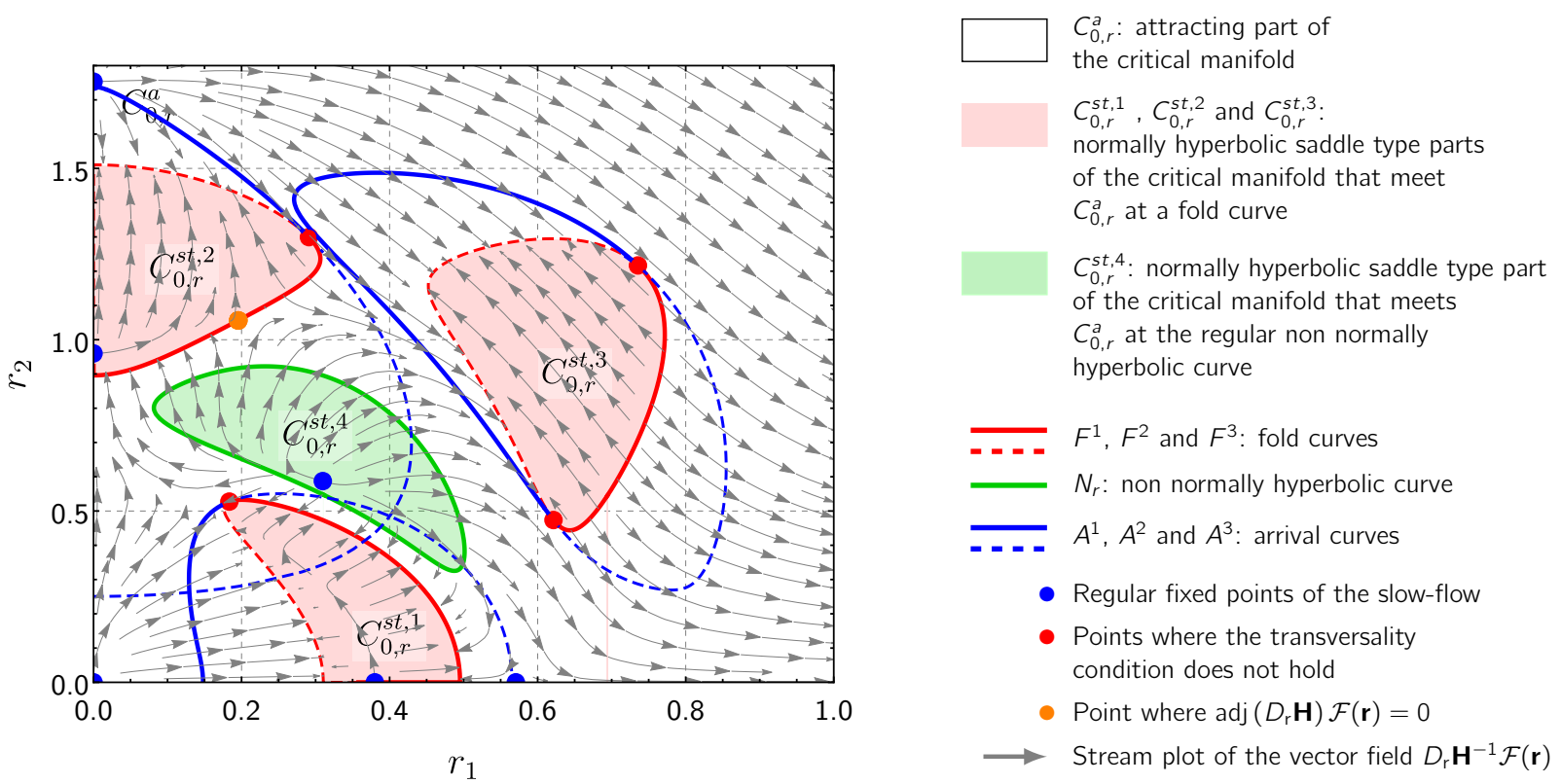

Figure 3: The different parts of the critical manifold $C_{0}$ are represented in the $\left(r_{1}, r_{2}\right)$-plane, as defined by (39): $C_{0, r}^{a}$ (white background) is a (regular) normally hyperbolic attracting part; $C_{0, r}^{s t, 1}$ (respectively $C_{0, r}^{s t, 2}$ and $C_{0, r}^{s t, 3}$ ) (salmon background) is a (regular) normally hyperbolic saddle type part that meets $C_{0, r}^{a}$ at a fold curve $F^{1}$ (respectively $F^{2}$ and $F^{3}$ ) (dashed and continuous red lines); $C_{0, r}^{s t, 4}$ (green background) is also a normally hyperbolic saddle type part which meets $C_{0, r}^{a}$ at the (regular) non normally hyperbolic curve $N_{r}$ (green line). The blue dashed lines $A_{1}^{1}$ (respectively $A_{1}^{2}$ and $A_{1}^{3}$ ) are the arrival curves associated with the red dashed lines $F_{1}^{1}$ (respectively $F_{1}^{2}$ and $F_{1}^{3}$ ) and the blue solid lines $A_{2}^{1}$ (respectively $A_{2}^{2}$ ) are the arrival curves associated with the red solid lines $F_{2}^{1}$ (respectively $F_{2}^{2}$ ). The stream plot of the vector field $D_{\mathbf{r}} \mathbf{H}^{-1} \mathcal{F}(\mathbf{r})$ is depicted with gray arrows. Blue dots represent the regular fixed points of the slow flow as defined by (43) (here unstable). The red dots are the points where the transversality condition (48) does not hold and the orange dot is the point where $\operatorname{adj}\left(D_{\mathbf{r}} \mathbf{H}\right) \mathcal{F}(\mathbf{r})=0$. Same parameters as in Fig. 2.

the variables $r_{1}$ and $r_{2}$ as

$$
D_{\mathbf{r}} \mathbf{H}(\mathbf{r}) \mathbf{r}^{\prime}=\mathcal{F}(\mathbf{r})
$$

where $\mathbf{r}=\left(r_{1}, r_{2}\right)^{T}$,

$$
D_{\mathbf{r}} \mathbf{H}(\mathbf{r})=\left(\begin{array}{ll}
\frac{\partial H_{1}}{\partial r_{1}}\left(r_{1}, r_{2}\right) & \frac{\partial H_{1}}{\partial r_{2}}\left(r_{1}, r_{2}\right) \\
\frac{\partial H_{2}}{\partial r_{1}}\left(r_{1}, r_{2}\right) & \frac{\partial H_{2}}{\partial r_{2}}\left(r_{1}, r_{2}\right)
\end{array}\right)
$$

is the Jacobian matrix of the function vector $\mathbf{H}(\mathbf{r})=\left(H_{1}(\mathbf{r}), H_{2}(\mathbf{r})\right)^{T}$ and

$$
\mathcal{F}(\mathbf{r})=\left(\begin{array}{c}
2 \sqrt{H_{1}\left(r_{1}, r_{2}\right)\left(a_{7}^{2}+a_{8}^{2}\right)} \\
2 \sqrt{H_{2}\left(r_{1}, r_{2}\right)\left(a_{9}^{2}+a_{10}^{2}\right)}
\end{array}\right) \mathbf{h}(\mathbf{r})
$$

In the above equation the vector function $\mathbf{h}(\mathbf{r})$ is obtained by replacing in $\mathbf{f}(\mathbf{s}, \mathbf{r})$ the variables $s_{1}, s_{2}, \Delta_{1}$ and $\Delta_{2}$ by their expressions as functions of $r_{1}$ and $r_{2}$.

From Eq. (40), it is possible to detect fixed points or equivalently regular fixed points as

$$
\begin{aligned}
\mathcal{F}(\mathbf{r}) & =\mathbf{0}, \\
\operatorname{det}\left(D_{\mathbf{r}} \mathbf{H}(\mathbf{r})\right) & \neq 0 .
\end{aligned}
$$

For the given nominal parameter values, the stream plot of the vector field $D_{\mathbf{r}} \mathbf{H}^{-1} \mathcal{F}(\mathbf{r})$ associated with the dynamical system (40) is depicted in Fig. 3 by gray arrows and the regular fixed points are reported 
as blue dots. A stability analysis has been performed given that all the fixed points are unstable. In the following magenta dots will be used to represent stable fixed points. A stable fixed point of the slow flow (19) characterizes a periodic regime of the initial system (5) if one of the variables $r_{i}(i=1,2)$ is equal to zero; otherwise the regime is quasi-periodic with two frequency components.

Fixed points no longer exist in situations where

$$
\begin{aligned}
\mathcal{F}(\mathbf{r}) & =\mathbf{0}, \\
\operatorname{det}\left(D_{\mathbf{r}} \mathbf{H}(\mathbf{r})\right) & =0 .
\end{aligned}
$$

The points in which Eqs. (44) hold are called folded singularities in the literature of slow-fast dynamical systems. Considering the relation

$$
\operatorname{det}\left(D_{\mathbf{r}} \mathbf{H}(\mathbf{r})\right)=-4 r_{1} r_{2} \operatorname{det}\left(D_{\mathbf{t}} \mathbf{g}_{0}(\mathbf{s}, \mathbf{t})\right)
$$

it follows that fold curves can be defined from $\operatorname{det}\left(D_{\mathbf{r}} \mathbf{H}(\mathbf{r})\right)=0$ and that folded singularities correspond to situations in which fixed points and fold points coincide. Folded singularities are hints of particular solutions of such slow-fast systems, called canard cycles [37]. These solutions are not investigated in this paper.

\subsection{Relaxation oscillations}

As suggested by the particular shape of the critical manifold, we examine in this section if specific solutions of the slow-fast system named relaxation oscillations can occur. These solutions characterize complicate motions named SMR of the mechanical system (5). We focus here on the limit case $\epsilon \rightarrow 0$. A relaxation oscillation is a solution consisting of successive fast and slow segments forming a closed loop in the $\left(r_{1}, r_{2}\right)$-plane representation of the critical manifold. The fast segments satisfy the fast subsystem (32) whereas the slow segments are obtained from the slow subsystem (31). Hence the slow segments take place on $C_{0}$ and the fast ones are outside of $C_{0}$. A switch from a slow segment to a fast one can occur when the trajectory crosses a fold line. In this case the fast segment appears as a jump from a fold point (in $C_{0}$ ) to an (arrival) point in $C_{0}$.

\subsubsection{Transversality condition}

The transversality condition is defined as a crossing condition with respect to a fold curve $F^{i}(i=1,2$ or 3 ) of the slow dynamics. It is obtained writing that the slow flow, projected in the $\left(r_{1}, r_{2}\right)$-plane, is not tangent to a fold curve $F^{i}(i=1,2$ or 3 ) (i.e. a jump can occur).

The slow subsystem (40) being by definition singular on the fold curve, it is first desingularized introducing the time rescaling $\tau_{1}=\tau / \operatorname{det}\left(D_{\mathbf{r}} \mathbf{H}(\mathbf{r})\right)$, which leads to

$$
\mathbf{r}^{\prime}=\operatorname{adj}\left(D_{\mathbf{r}}(\mathbf{r}) \mathbf{H}\right) \mathcal{F}(\mathbf{r})
$$

where $\operatorname{adj}($.$) denotes the adjoint matrix of (.) and ()^{\prime}$ is now the derivative with respect to the new time $\tau_{1}$.

Recalling that a fold curve in the $\left(r_{1}, r_{2}\right)$-plane is defined as $\operatorname{det}\left(D_{\mathbf{r}} \mathbf{H}(\mathbf{r})\right)=0$ (see Sect. 3.4), the transversality condition at a point $\mathbf{r}$ on a fold curve $F^{i}(i=1,2$ or 3$)$ is written as follows

$$
\operatorname{grad}_{\mathbf{r}}\left(\operatorname{det}\left(D_{\mathbf{r}} \mathbf{H}(\mathbf{r})\right)\right) \cdot\left[\operatorname{adj}\left(D_{\mathbf{r}} \mathbf{H}\right) \mathcal{F}(\mathbf{r})\right] \neq 0
$$

where $\operatorname{grad}_{\mathbf{r}}($.$) denotes the gradient of (.) with respect to \mathbf{r}$ and $\cdot$ denotes the usual dot product in $\mathbb{R}^{2}$. The points where the transversality condition does not hold are obtained solving

$$
\operatorname{grad}_{\mathbf{r}}\left(\operatorname{det}\left(D_{\mathbf{r}} \mathbf{H}(\mathbf{r})\right)\right) \cdot\left[\operatorname{adj}\left(D_{\mathbf{r}} \mathbf{H}\right) \mathcal{F}(\mathbf{r})\right]=0
$$

for $\operatorname{adj}\left(D_{\mathbf{r}} \mathbf{H}\right) \mathcal{F}(\mathbf{r}) \neq 0$. The points defined by (48) are depicted in red on Fig. 3 for the given nominal parameter values. These points are isolated and divide the fold curves into two parts

$$
F^{i}=F_{1}^{i} \cup F_{2}^{i} .
$$


In Fig. 3 the red dashed (respectively solid) lines are arbitrarily chosen to depict $F_{1}^{1}, F_{1}^{2}$ and $F_{1}^{3}$ (respectively $F_{2}^{1}, F_{2}^{2}$ and $F_{2}^{3}$ ). Moreover, we can see that the stream plot of the vector field $D_{\mathbf{r}} \mathbf{H}^{-1} \mathcal{F}(\mathbf{r})$ can head towards or against the fold lines.

If $\operatorname{adj}\left(D_{\mathbf{r}} \mathbf{H}\right) \mathcal{F}(\mathbf{r})=0$, the considered points are no longer points where the transversality condition does not hold, but fixed points of the desingularized system (46), which are also hints of possible canard cycles. In Fig. 3, a point, depicted as an orange dot, corresponds to this situation.

\subsubsection{Jumps and arrival curves}

When the slow dynamics described by (40) reaches a point $\mathbf{r}^{J}=\left(r_{1}^{J}, r_{2}^{J}\right)$ of a part $F_{j}^{i}$ (with $i=1,2,3$ and $j=1,2$ ) of the fold curve $F^{i}$ (this can occur if the stream plot of the vector field $D_{\mathbf{r}} \mathbf{H}^{-1} \mathcal{F}(\mathbf{r})$ heads towards $F_{j}^{i}$ ), the trajectory can leave the critical manifold $C_{0}$ (and the slow epoch) and undergoes a jump (a fast episode) before reaching another part of $C_{0, r}^{a}$. The point $\mathbf{r}^{J}$ is named jump point. The jump is described by the fast subsystem (32) with the jump point $\mathbf{r}^{J}$ as initial condition. Hence the possible drop point, denoted as $\mathbf{r}^{D}=\left(r_{1}^{D}, r_{2}^{D}\right)$, of the fast dynamics on $C_{0, r}^{a}$ is determined as a fixed point of the fast subsystem (32), that is

$$
\mathbf{g}_{0}\left(\mathbf{s}^{J}, \mathbf{t}\right)=0
$$

where $\mathbf{s}^{J}$ is obtained from Eqs. (36) evaluated at $\mathbf{r}^{J}$.

Manipulating Eq. (50), it can be shown that the drop point $\mathbf{r}^{D}$ is defined as the root of the following set of equations

$$
\begin{aligned}
& H_{1}\left(r_{1}^{D}, r_{2}^{D}\right)=H_{1}\left(r_{1}^{J}, r_{2}^{J}\right) \\
& H_{2}\left(r_{1}^{D}, r_{2}^{D}\right)=H_{2}\left(r_{1}^{J}, r_{2}^{J}\right),
\end{aligned}
$$

where the functions $H_{1}$ and $H_{2}$ are defined by Eqs. (37). The solutions obtained solving Eqs. (51) for each point of $F_{j}^{i}$ define the arrival curve denoted as $A_{j}^{i}$. The arrival curves are also reported in Fig. 3 . The blue dashed line $A_{1}^{1}$ (respectively $A_{1}^{2}$ and $A_{1}^{3}$ ) is the arrival curve associated with the red dashed line $F_{1}^{1}$ (respectively $F_{1}^{2}$ and $F_{1}^{3}$ ) and the blue solid line $A_{2}^{1}$ (respectively $A_{2}^{2}$ ) is the arrival curve associated with the red solid line $F_{2}^{1}$ (respectively $F_{2}^{2}$ ).

The arrival curve (or a part of it) is stable, and therefore potentially reached by the trajectory at the end of a fast jump, if it is located on $C_{0, r}^{a}$, and unstable if it is located on $C_{0, r}^{s t, i}(i=1,2,3$ or 4$)$.

\subsubsection{Description of a possible relaxation oscillation scenario}

The previous results, leading to the general form of the critical manifold depicted in Figs. 2 and 3, allow us to predict different possible scenarios of relaxation oscillations. The latter can be defined from elementary relaxation oscillations.

An elementary relaxation oscillation is defined as a succession of fast, slow, fast and slow epochs starting from and dropping on the same part $F_{j}^{i}$. This can for example occur in Fig. 3, considering the case where the slow dynamics in $C_{0, r}^{a}$ leads the trajectory to a jump point $P_{1}$ of $F_{1}^{1}$. Next a fast jump occurs before reaching a drop point $P_{2}$ of $A_{1}^{1}$. Then, from $P_{2}$, the slow dynamics reaches the fold curve $F_{2}^{1}$ at the jump point $P_{3}$, followed by a jump to a drop point $P_{4}$ of $A_{2}^{1}$. Finally the slow dynamics reaches the fold curve $F_{1}^{1}$ at a jump point $P_{5}$. If $P_{1}=P_{5}$, the elementary relaxation oscillation defines a (sustained) simple relaxation oscillation.

In the case of Fig. 3, it is easy to verify that elementary relaxation oscillations can occur in the neighborhood of $C_{0, r}^{s t, 1}$ and $C_{0, r}^{s t, 2}$, but not in the neighborhood of $C_{0, r}^{s t, 3}$. Indeed, a necessary condition to observe relaxation oscillations on both sides of a normally hyperbolic saddle type part $C_{0, r}^{s t, i}(i=1,2,3)$ of the critical manifold is that both $F_{1}^{i}$ and $F_{2}^{i}$ contain parts where the vector field $D_{\mathbf{r}} \mathbf{H}^{-1} \mathcal{F}(\mathbf{r})$ is incoming.

Unlike the case where the slow dynamics can be described in a one-dimensional space, here the critical manifold $C_{0}$ appears as a two-dimensional parametric surface. Consequently the condition $P_{1}=P_{5}$ is not always satisfied. Moreover a simple relaxation oscillation can appear after a transient phase including successive elementary relaxation oscillations. We have not developed a method to characterize the existence of such simple relaxation oscillations. 
More complex (sustained) relaxation oscillation scenarios may be considered, such as a double relaxation oscillation which is defined from two successive elementary relaxation oscillations occurring alternatively in the neighborhoods of two different $C_{0, r}^{s t, i}$.

The next section provides an overview of the possible steady-states regimes by means of numerical simulations and aims at understanding them with regard to the theoretical results presented in this section. Note that basins of attraction of the possible steady-states regimes must be considered besides the previous local analysis. Indeed, for a given set of parameters, the system may have several stable attractors (stable fixed points or sustained relaxation oscillations) and the initial conditions determine which one is reached.

\section{Numerical results}

In this part, numerical simulations are performed both to validate and to complete the asymptotic analysis presented in Sect. 3 by means of direct numerical integration of the slow flow (19) and of the initial system (12). For the numerical simulations, we fix $\epsilon=0.01$ and the other parameters are always chosen to ensure that the first two modes of the primary system (3a-3d) undergo Hopf bifurcations at $\gamma=\gamma_{\text {Hopf,1 }}$ and $\gamma=\gamma_{\text {Hopf,2 }}$ respectively (i.e. the real parts $\rho_{1}$ and $\rho_{2}$ (see Eq. (9)) of the eigenvalues become greater than zero at $\gamma_{\text {Hopf,1 }}$ and $\gamma_{\text {Hopf,2 }}$ respectively). The numerical values of $\gamma_{\text {Hopf,1 }}$ and $\gamma_{\text {Hopf,2 }}$ are given for each situation under study in the following sections.

In all the examples presented below, the friction coefficient $\gamma$ is chosen such that $\gamma_{\text {Hopf, } 1}<\gamma_{\text {Hopf, } 2}<\gamma$. This choice validates the use of the reduced model (13) and the slow-flow formulation where $s_{1}$ and $s_{2}$ represent the two unstable modes of the primary system and $r_{1}$ and $r_{2}$ represent the frequency components (related to the two unstable modes of the primary system) of the relative displacement between the NES and the primary system.

Note that the primary structure (without NES) is linear, consequently it undergoes, when it is unstable, an exponential growth (i.e. the solution is not bounded). Therefore, all the responses of the coupled system shown in this section, which show bounded amplitudes, correspond to a quench of the "double-instability" by the single NES. These bounded motions can occur through periodic responses, quasi-periodic responses or complex SMR. Another type of response is shown at the end of the section (Sect 4.4).

Sect. 4.1 investigates the competition between two stable fixed points of the slow flow corresponding to the competition between two periodic or quasi-periodic regimes for the mechanical system; Sect. 4.2 analyzes the competition between a sustained relaxation oscillation and a stable fixed point of the slow flow corresponding to the competition between an SMR and a periodic or quasi-periodic regime; in Sect. 4.3 the competition between two kinds of sustained relaxation oscillations, corresponding to the competition between two SMR, is addressed. Finally, the case of the bifurcation of a fixed point to a limit cycle of the slow subsystem, corresponding to another type of amplitude and phase modulated response of the mechanical system, is presented in Sect. 4.4 .

\subsection{Competition between two stable fixed points of the slow flow}

In this section the following set of parameters is used:

$$
\begin{aligned}
\eta_{1} & =0.01 & \eta_{2} & =0.07 & \eta_{3}=0.01 & \eta_{4}=0.07 \\
\Omega_{2} & =0.93 & \Omega_{3} & =1 & \Omega_{4}=1.7 & \\
\eta_{a} & =0.01 & \Omega_{a} & =0.95 & & \\
\sigma & =1 & \gamma & =0.09075 & & \\
\mu & =0.395 & \alpha & =7 . & &
\end{aligned}
$$

For this set of parameters, $\gamma_{\mathrm{Hopf}, 1}=0.081, \gamma_{\mathrm{Hopf}, 2}=0.088$ and the invariant manifold $C_{0}$ admits a decomposition in the form of (39). The fixed points of the slow flow are obtained solving Eq. (43), which gives four unstable fixed points including the trivial point $(0,0)$, and two stable fixed points, denoted as $\mathbf{r}_{s, 1}^{*}$ and $\mathbf{r}_{s, 2}^{*}$, whose coordinates in the $\left(r_{1}, r_{2}\right)$-plane are respectively

$$
\mathbf{r}_{s, 1}^{*}=(0.26,0) \quad \text { and } \quad \mathbf{r}_{s, 2}^{*}=(0,0.45) .
$$


The two stable fixed points of the slow flow correspond to a mitigation of the two unstable modes of the primary system. Note that if $\mathbf{r}_{s, 1}^{*}$ is reached, the first unstable mode is mitigated through a periodic response of the physical system $(12)^{1}$ whereas the second unstable mode is completely suppressed. If $\mathbf{r}_{s, 2}^{*}$ is reached, the first unstable mode is completely suppressed whereas the second unstable mode is mitigated through a periodic response. The associated critical manifold $C_{0}$ is depicted on Fig. 4(b) and Fig. 5(b). The stable fixed points are represented by magenta dots and the unstable fixed points by blue dots. Two stable and one unstable fixed points are in the neighborhood of the trivial point $(0,0)$. These four fixed points are on the (regular) normally hyperbolic attracting part $C_{0, r}^{a}$. The other two fixed points are far from $(0,0)$. The stream plot of the vector field of Eq. (40), also reported in Fig. 4(b) and Fig. 5(b), suggests that each stable fixed point admits a basin of attraction in the neighborhood of the trivial point $(0,0)$.

The direct numerical integration of the slow flow (19) is performed to localize, on the critical manifold $C_{0}$, the basin of attraction of each stable fixed point.

The time series of $s_{1}, s_{2}, r_{1}$ and $r_{2}$ obtained from two different initial conditions $\mathbf{r}_{0,1}=\left(r_{1}(0), r_{2}(0)\right)=$ $(0.02,0.1)$ and $\mathbf{r}_{0,2}=(0.02,0.2)$ are reported respectively on Fig. $4(\mathrm{a})$ and Fig. $5(\mathrm{a})$, and the corresponding trajectories in the $\left(r_{1}, r_{2}\right)$-plane are plotted on Fig. 4(b) and Fig. 5(b). In the latter, the initial condition points $\mathbf{r}_{0,1}$ and $\mathbf{r}_{0,2}$ are represented by light blue dots and the trajectories of the slow flow are depicted using a color gradient from light blue at $t=0$ to black at $t=30000$. These trajectories give a good idea of the basins of attraction and of the role played by the unstable fixed points. The basin of attraction of $\mathbf{r}_{s, 1}^{*}$ is located between the $r_{1}$-axis and the streamline that links the trivial point to the unstable fixed point located at $(0.16,0.25)$; the basin of attraction of $\mathbf{r}_{s, 2}^{*}$ is located between the same streamline and the $r_{2}$-axis.

Finally, the time series obtained solving the complete equations (12) are also reported in Fig. 4(a) and Fig. 5(a) following the remarks below.

From Eqs. (14) to (16) and (18), the variables of the complete model (12) can be expressed as functions of the slow-flow variables as follows

$$
\begin{aligned}
& q_{1}=s_{1} e^{j\left(\lambda_{1}^{I} t+\delta_{1}\right)}, \quad q_{2}=s_{2} e^{j\left(\lambda_{2}^{I} t+\delta_{1}\right)}, \\
& v_{1}=\frac{r_{1}}{\lambda_{1}^{I}} \sin \left(\lambda_{1}^{I} t+\theta_{1}\right)=A_{v_{1}} \sin \left(\lambda_{1}^{I} t+\theta_{1}\right), \quad v_{2}=\frac{r_{2}}{\lambda_{2}^{I}} \sin \left(\lambda_{2}^{I} t+\theta_{2}\right)=A_{v_{2}} \sin \left(\lambda_{2}^{I} t+\theta_{2}\right) .
\end{aligned}
$$

To analyze the results, the modulus of the variables $q_{1}$ and $q_{2}$ (orange lines) of the system (12) must be compared to the variables $s_{1}$ and $s_{2}$ (blue lines) of the slow flow (19) respectively. Moreover, the spectrograms of the signal $v(t)$ (resulting from the numerical integration of Eq. (12)) are then computed and the amplitudes $A_{v_{1}}$ and $A_{v_{2}}$ of the components $v_{1}(t)$ and $v_{2}(t)$ related to the frequencies $\lambda_{1}^{I}$ and $\lambda_{2}^{I}$ respectively are extracted. The variables $\lambda_{1}^{I} A_{v_{1}}$ and $\lambda_{2}^{I} A_{v_{2}}$ (black dashed lines) are to be compared to the variables $r_{1}$ and $r_{2}$ (green lines) of the slow flow (19).

In each case the behavior of $s_{1}$ (respectively $r_{1}, s_{2}$ and $r_{2}$ ) is found close to the behavior of $q_{1}$ (respectively $\lambda_{1}^{I} A_{v_{1}}, q_{2}$ and $\lambda_{2}^{I} A_{v_{2}}$ ), which allows us to validate the theoretical development presented in Section 3.

Even if the previous comparisons show a good agreement, quantitative differences are nevertheless observed. We recall that two steps separate the initial mechanical system (12) and its approximated slow flow (19): the reduction of the dynamics keeping only the unstable modes and the averaging with respect to the frequencies of these two unstable modes. Additional numerical simulations (not displayed here) show that the quantitative differences observed here (and also in the following sections) are mainly due to the averaging step.

\footnotetext{
${ }^{1}$ Indeed, if $r_{1}=0$ or $r_{2}=0$, the non averaged initial system undergoes periodic oscillations.
} 


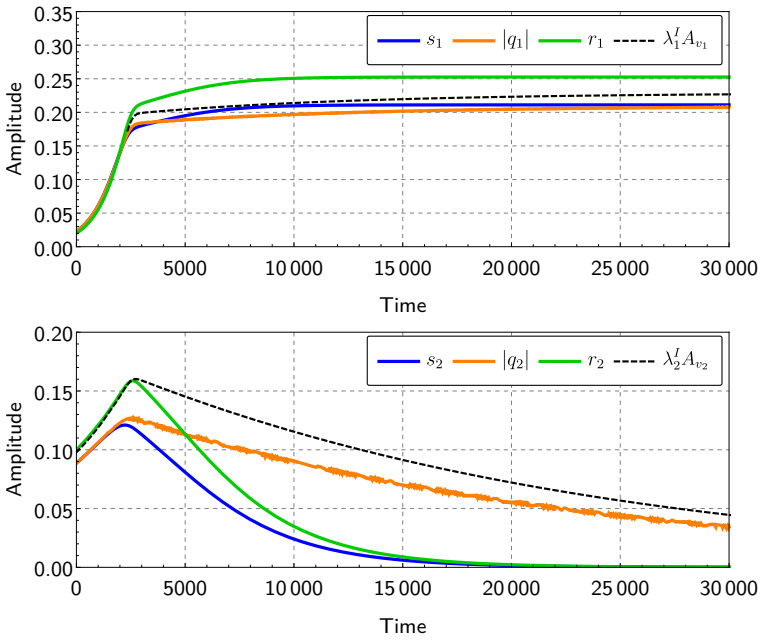

(a)

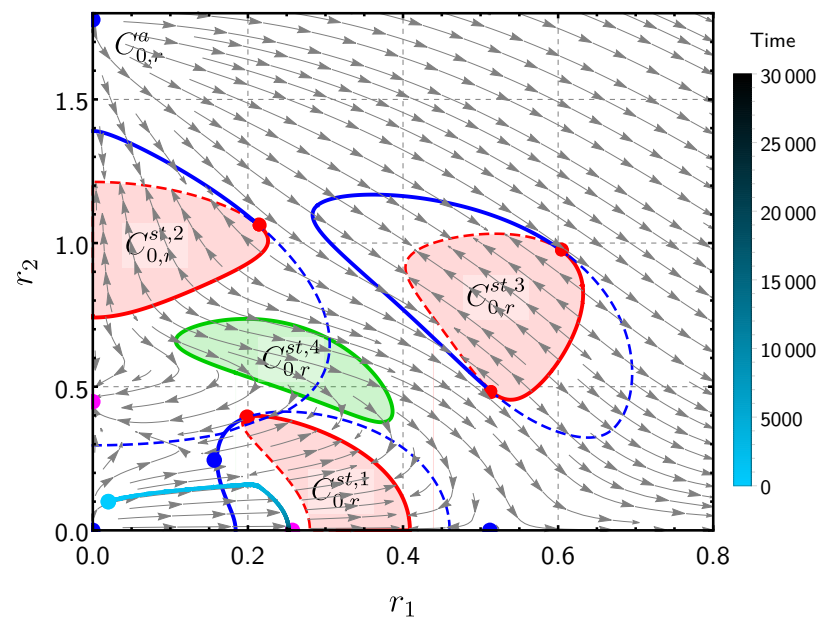

(b)

Figure 4: Competition between two stable fixed points of the slow flow (1 $1^{\text {st }}$ example). (a) Amplitudes $r_{1}, s_{1}$ (top) and $r_{2}, s_{2}$ (bottom) as functions of time with the initial condition $\mathbf{r}_{0,1}=(0.02,0.1)$ obtained by solving numerically the slow flow (19). The time series of $q_{1}$ (top) and $q_{2}$ (bottom) obtained solving numerically Eqs. (12) are also shown. (b) Similar representation as in Fig. 3 with the addition of the trajectories (solid lines with a color gradient from light blue at $t=0$ to black at $t=30000$ ) shown in (a); the magenta dots are the stable fixed points of the slow flow. The parameters (52) are used.

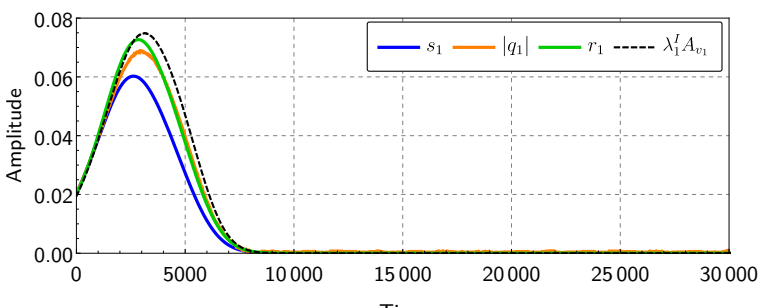

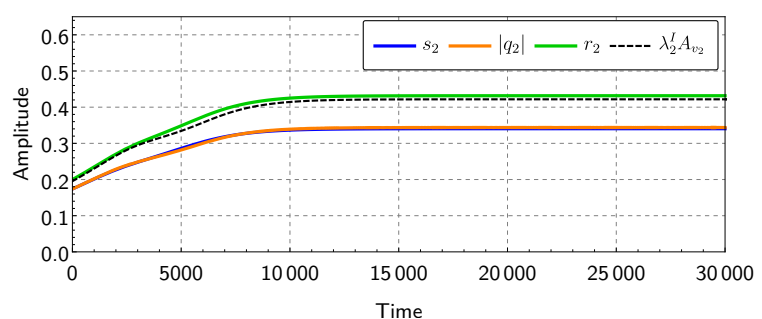

(a)

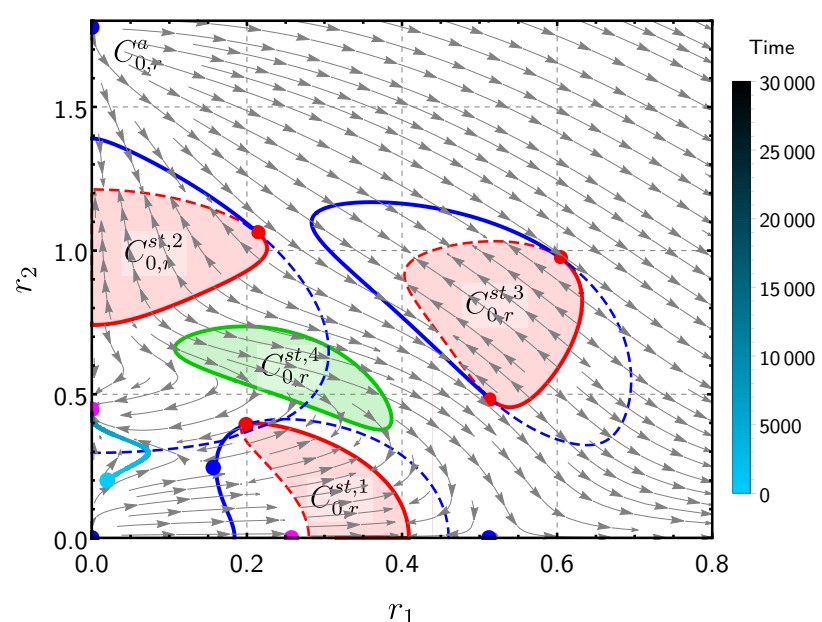

(b)

Figure 5: Competition between two stable fixed points of the slow flow ( $2^{\text {nd }}$ example). Same caption as in Fig. 4 with the initial condition $\mathbf{r}_{0,2}=(0.02,0.2)$. 


\subsection{Competition between a sustained relaxation oscillation and a stable fixed point of the slow flow}

To illustrate the competition between sustained relaxation oscillations and a stable fixed point we use the following parameters

$$
\begin{aligned}
& \eta_{2}=0.07 \\
& \Omega_{2}=0.93 \\
& \Omega_{3}=1 \\
& \eta_{a}=0.01 \\
& \Omega_{a}=0.95 \\
& \sigma=1 \\
& \gamma=0.09 \\
& \eta_{3}=0.01 \\
& \eta_{4}=0.07 \\
& \Omega_{4}=1.7 \\
& \mu=0.25 \\
& \alpha=7 \text {. }
\end{aligned}
$$

The parameters of the primary system are here unchanged compared to those of Sect. 4.1 (only the bifurcation parameter $\gamma$ and the NES parameters $\mu$ ans $\alpha$ are modified). Therefore, the values of the Hopf bifurcation points are still $\gamma_{\mathrm{Hopf}, 1}=0.081$ and $\gamma_{\mathrm{Hopf}, 2}=0.088$. Moreover, the invariant manifold $C_{0}$ admits again a decomposition in the form of (39); the slow flow now admits six fixed points, among which only one is stable. The latter, denoted as $\mathbf{r}_{s, 3}^{*}$, has the following coordinates in the $\left(r_{1}, r_{2}\right)$-plane:

$$
\mathbf{r}_{s, 3}^{*}=(0,0.57)
$$

If $\mathbf{r}_{s, 3}^{*}$ is reached, only the first unstable mode is completely suppressed whereas the second unstable mode is mitigated through a periodic response. The critical manifold $C_{0}$ is reported in Fig. 6(b) and Fig. 7(b) where the stable fixed point is again represented by a magenta dot and the unstable points by blue dots. The stable fixed point and two unstable fixed points (excluding the trivial point) are in the neighborhood of the trivial point $(0,0)$, the other unstable points being on the contrary far from $(0,0)$. The stream plot of the vector field of Eq. (40) suggests that, depending of the initial condition point, the system can reach the stable fixed point $\mathbf{r}_{s, 3}^{*}$ or initiate another type of oscillations.

Direct numerical integration of the slow flow (19) is performed to localize, on the critical manifold $C_{0}$, the basin of attraction of the stable fixed point and to characterize the other type of oscillations. The time series obtained from the slow flow (19) considering the initial condition $\mathbf{r}_{0,3}=(0.01,0.15)$ are plotted in Fig. 6 (a) and, using a color gradient, the corresponding trajectory in the $\left(r_{1}, r_{2}\right)$-plane is depicted in Fig. 6(b). It can be seen that the stable fixed point is reached.

Results related to a second initial condition point $\mathbf{r}_{0,4}=(0.01,0.03)$ are reported in Fig. 7. Again, the time series are plotted in Fig. $7(\mathrm{a})$ and the trajectory in the $\left(r_{1}, r_{2}\right)$-plane in Fig. $7(\mathrm{~b})$. To better understand the observed behavior, three-dimensional plots are also used (see Figs. 7(c) and 7(d)), where the trajectories are plotted in the $\left(r_{1}, r_{2}, s_{1}\right)$-space and the $\left(r_{1}, r_{2}, s_{2}\right)$-space respectively and superimposed to the surfaces, defined by Eqs. (36), characterizing the invariant manifold $C_{0}$. Moreover, using the same color code as in Fig. 3, the fold and arrival curves as well as the points where the transversality condition is lost are depicted in Figs. $7(\mathrm{c})$ and $7(\mathrm{~d})$.

Fig. 7(b) shows that after transient elementary relaxation oscillations (described in Sect. 3.5.3) with respect to the normally hyperbolic saddle type part $C_{0, r}^{s t, 1}$ of the critical manifold, a sustained simple relaxation oscillation (described in Sect. 3.5.3) occurs along the straight line $r_{2}=0\left(s_{2}\right.$ and $r_{2}$ tend towards zero as visible in Fig. 7(a)). Observing Figs. 7(c) and 7(d), we can see that this simple relaxation oscillation is due to an $S$-shape of the critical manifold in the $\left(r_{1}, r_{2}, s_{1}\right)$-space and in the plane $r_{2}=0$. This means that the first unstable mode here is completely suppressed while the second unstable mode is mitigated through a sustained simple relaxation oscillation which corresponds to an SMR for the initial physical system (12).

As in the previous case studied in Sect. 4.1, the boundary between the basins of attractions of the two attractors appears to be the connection between two unstable fixed points, i.e. the trivial fixed point and the fixed point located at $(0.22,0.18)$.

Here again, the predictions obtained with the slow flow (19) are in good agreement with the simulations of the complete system (12) (see Figs. 6(a) and 7(a)). 


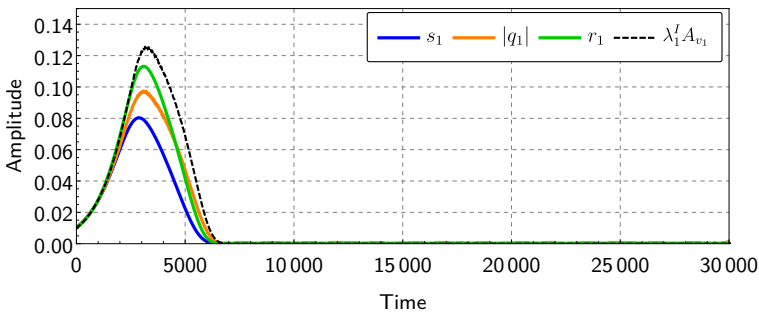

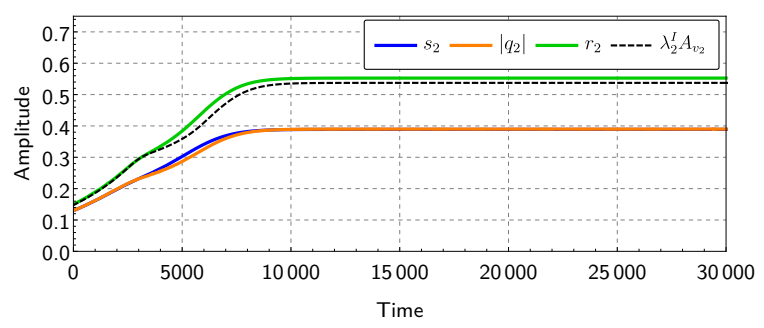

(a)

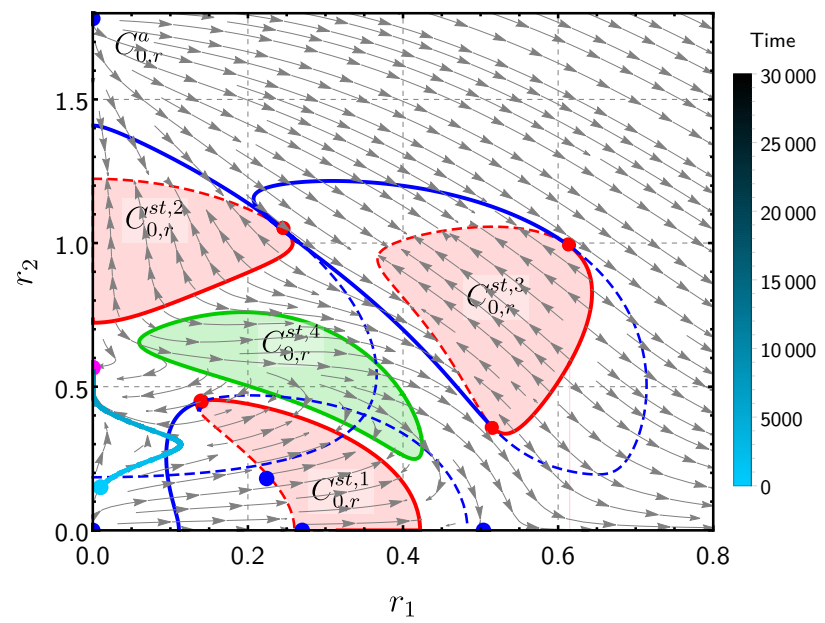

(b)

Figure 6: Competition between a sustained relaxation oscillation and a stable fixed point of the slow flow (1 $1^{\text {st }}$ example). Same caption as in Fig. 4 with the initial condition $\mathbf{r}_{0,3}=(0.01,0.15)$. The set of parameters (56) is used.

\subsection{Competition between two kinds of sustained relaxation oscillations}

In this section the following parameters are used

$$
\begin{aligned}
& \begin{aligned}
\eta_{1}=0.01 & \eta_{2}=0.07 & \eta_{3}=0.01 & \eta_{4}=0.07 \\
\Omega_{2}=0.93 & \Omega_{3}=1 & \Omega_{4}=1.7 &
\end{aligned} \\
& \eta_{a}=0.01 \quad \Omega_{a}=0.95 \\
& \sigma=0.85 \quad \gamma=0.0935 \\
& \mu=0.28 \quad \alpha=5
\end{aligned}
$$

for which the slow flow (19) has six fixed points, all unstable. The values of the Hopf bifurcation points are $\gamma_{\text {Hopf, } 1}=0.078$ and $\gamma_{\text {Hopf, } 2}=0.088$. The critical manifold $C_{0}$ is reported in Fig. $8(\mathrm{~b})$ and Fig. $9(\mathrm{~b})$. Note that there are no unstable fixed points in the neighborhood of the trivial point. Obviously in this case, because the slow flow has no stable fixed point, the instability mitigation cannot be performed through quasi-periodic or periodic regimes.

In practice, depending on the chosen initial conditions, two kinds of sustained relaxation oscillations can be observed by means of numerical simulations of the slow flow (19).

Fig. 8 illustrates the first scenario. The time series are obtained from the initial condition $\mathbf{r}_{0,5}=$ $(0.05,0.05)$ and are reported in Fig. 8(a). This scenario is similar to that observed in Sect. 4.2 and reported in Fig. 7: after transient elementary relaxation oscillations, the second unstable mode is completely suppressed (see Fig. 8(a)) whereas the first unstable mode is mitigated through a sustained simple relaxation oscillation taking place along the straight line $r_{2}=0$ (see the black curve in Fig. 8(c)). This simple relaxation oscillation is due to the $S$-shape of the critical manifold in the $\left(r_{1}, r_{2}, s_{1}\right)$-space and in the plane $r_{2}=0$. It consists of four parts: two fast jumps (from the fold curve $F_{1}^{1}$ to the arrival curve $A_{1}^{1}$ and from the fold curve $F_{2}^{1}$ to the arrival curve $A_{2}^{1}$ ) and two slow epochs on $C_{0, r}^{a}$.

Fig. 9 illustrates the second scenario. The time series are obtained from the initial condition $\mathbf{r}_{0,6}=$ $(0.1,0.1)$ and are reported in Fig. 9(a). After a quick transient phase, the two unstable modes are mitigated through complex relaxation oscillations with respect to the normally hyperbolic saddle type parts $C_{0, r}^{s t, 1}$ and $C_{0, r}^{s t, 2}$ of the critical manifold successively. The complex relaxation oscillations here consist of three parts: an elementary relaxation oscillation due to the $S$-shape of the critical manifold in the $\left(r_{1}, r_{2}, s_{2}\right)$-space (see 


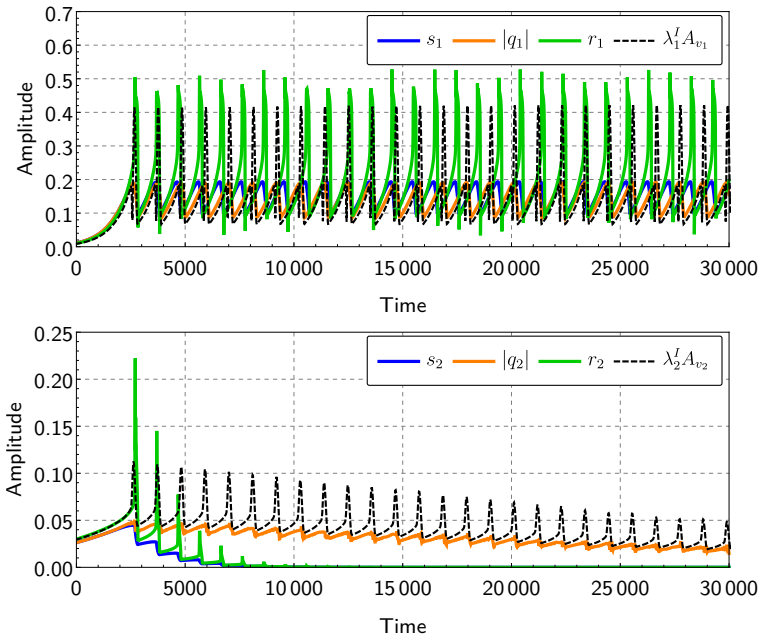

(a)

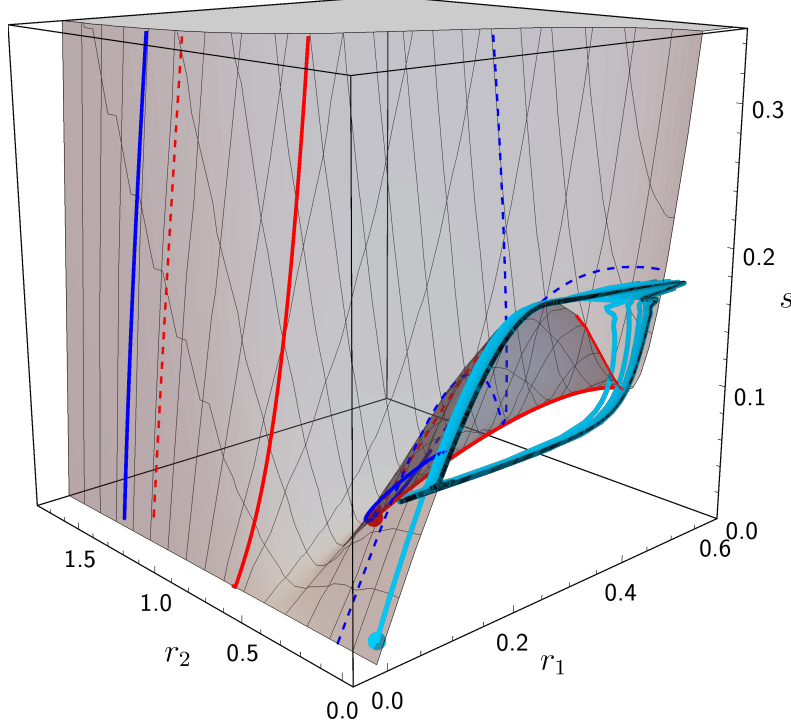

(c)

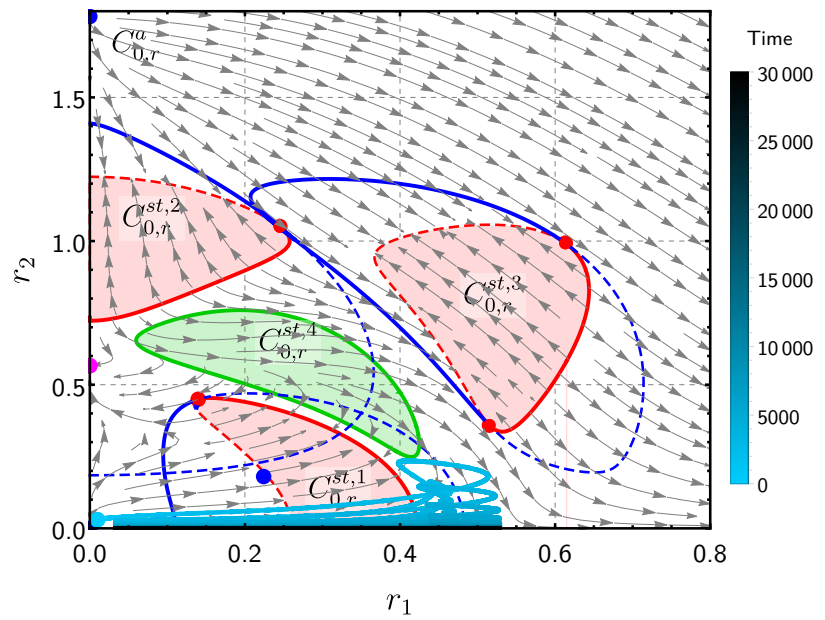

(b)

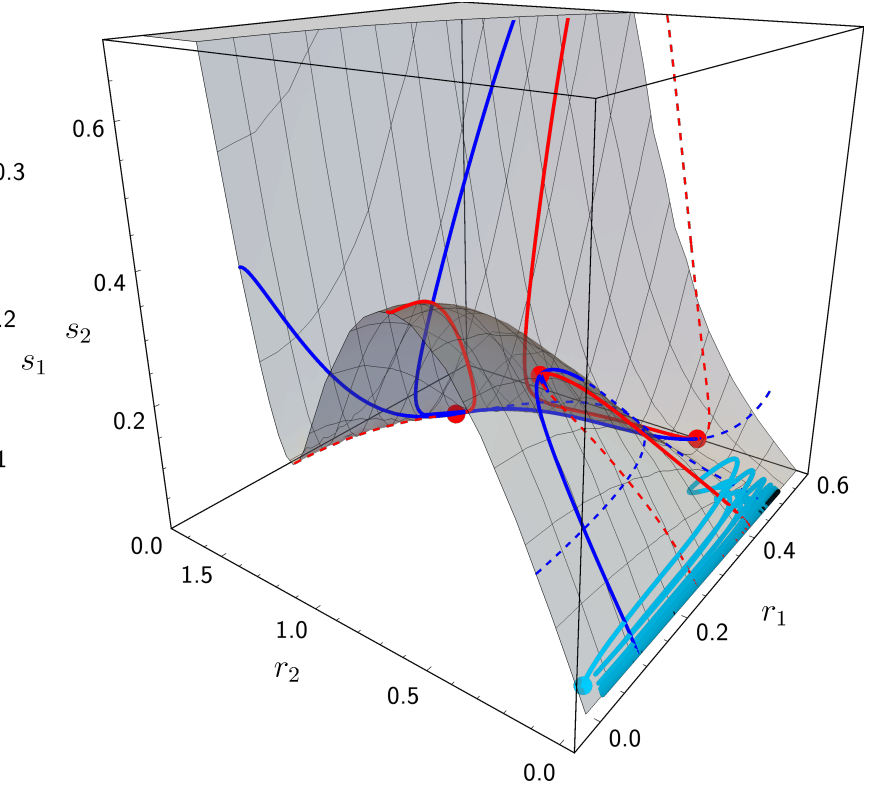

(d)

Figure 7: Competition between a sustained relaxation oscillation and a stable fixed point of the slow flow (2nd example). (a) and (b) Same captions as in Fig. 4 with the initial condition $\mathbf{r}_{0,4}=(0.01,0.03)$. In (c) and (d) the trajectory of the slow flow obtained from the numerical simulation of Eq. (19) is plotted in the $\left(r_{1}, r_{2}, s_{1}\right)$-space and the $\left(r_{1}, r_{2}, s_{2}\right)$-space respectively and superimposed to the surfaces given by Eqs. (37a) and (37b). The set of parameters (56) is used.

Fig. 9(d)), an elementary relaxation oscillation due to the $S$-shape of the critical manifold in the $\left(r_{1}, r_{2}, s_{1}\right)$ space (see Fig. 9(c)) and transient bursting oscillations (see Figs. 9(b) and 9(c)). This sustained complex relaxation oscillation regime is due to successive targeted energy transfers, first from the first unstable mode to the NES (principally when the jumps from $F_{1}^{2}$ to $A_{1}^{2}$ and from $F_{2}^{2}$ to $A_{2}^{2}$ occur) and then from the second mode to the NES (when the jumps from $F_{1}^{1}$ to $A_{1}^{1}$ occur). Note that those energy transfers do not occur simultaneously as shown in Fig. 9(a).

From [36], bursting oscillations are defined as patterns of time series alternating between near steadystate and rapid oscillatory phases. Here, the steady-state phases are the slow evolution parts on $C_{0, r}^{a}$ of the 
relaxation oscillation cycles and the rapid oscillatory phases correspond to the fast jumps which are now oscillating. This phenomenon is related to the normally hyperbolic saddle type part of $C^{0}, C_{0, r}^{s t, 4}$, that meets $C_{0, r}^{a}$ at the (regular) non normally hyperbolic curve $N_{r}$ (see Fig. 9(b)). Indeed, when a part of the arrival curve is in this area, it is made up of unstable fixed points of the fast subsystem (32). In this case, the attractor of the fast dynamics during a jump, at constant values of $s_{1}$ and $s_{2}$ (see Eq. (32a)), is no longer an equilibrium point but a limit cycle oscillation (LCO). Then, when the jump is finished, because $s_{1}$ and $s_{2}$ vary again the system leaves $C_{0, r}^{s t, 4}$ and the LCO undergoes a bifurcation to a new steady-state (slow) phase. Here the bursting oscillations are transient but depending on the parameter values, they can also been sustained. This scenario has not been studied in this paper.

The boundary between the basins of the two attractors does not appear as clearly as in the previous situations where it corresponded to the connection between two unstable points. Indeed, as noticed above, there are no unstable fixed points in the neighborhood of the trivial point. Here, the boundary depends on the first reached point on the fold curve $F_{1}$ and on the direction of the transient relaxation oscillations along $C_{0, r}^{s t, 1}$, which can move downward (first scenario) or upward (second scenario).

Finally, here also, the predictions obtained with the slow flow (19) are in good agreement with the complete system (12) (see Figs. 8(a) and 9(a)).

\subsection{Bifurcation of a fixed point to a limit cycle of the slow subsystem}

For this last example, the following parameters are used with two different values of the friction coefficient

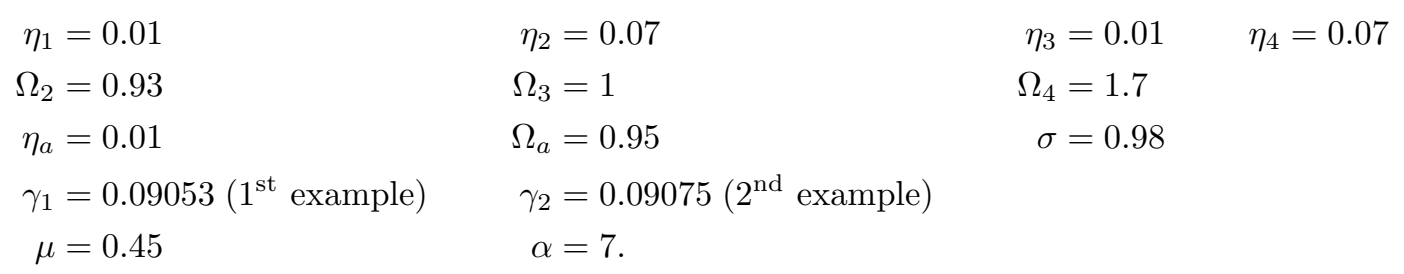

With the parameters (59), the primary system undergoes Hopf bifurcations at $\gamma_{\mathrm{Hopf}, 1}=0.08$ and $\gamma_{\mathrm{Hopf}, 2}=$ 0.086. The associated critical manifolds $C_{0}$ are reported in Fig. 10(b) and Fig. 10(d) for $\gamma=0.09053$ and $\gamma_{2}=0.09075$ respectively.

For $\gamma_{1}=0.09053$, the slow flow (19) admits a single stable fixed point having the following coordinates in the $\left(r_{1}, r_{2}\right)$-plane:

$$
\mathbf{r}_{s, 4}^{*}=(0.06,0.61)
$$

and five unstable fixed points. Note that if $\mathbf{r}_{s, 4}^{*}$ is reached, both unstable modes are mitigated through a periodic response of the physical system which therefore undergoes a quasi-periodic regime even if the amplitude of the component corresponding to the first unstable mode is much smaller than that of the second unstable mode. Numerical simulations of the slow flow (19) corresponding to this situation are shown in Figs. 10(a) and 10(b).

Then, when $\gamma$ increases, the stable fixed point $\mathbf{r}_{s, 4}^{*}$ of the slow flow undergoes a Hopf bifurcation and becomes unstable. For example, for $\gamma=0.09075$, the slow flow has only unstable fixed points, four of which are in the neighborhood of $(0,0)$.

Numerical simulations of the slow flow (19) corresponding to this second case have been performed for different values of the initial conditions, showing that only one stable attractor is present whose basin of attraction includes a neighborhood of the trivial point $(0,0)$ (as it was previously the case for the stable fixed point). The time series obtained from the initial condition $r_{0,7}=(0.05,0.05)$ are reported in Figs. 10(c) and Fig. 10(d). The figures show that after the bifurcation of the stable fixed point (60), the slow flow undergoes an LCO (see the black curve in Fig. 10(d)) which takes place in the attractive part $C_{0, r}^{a}$ of the critical manifold, and therefore only at the slow-time scale (there are no fast jumps, unlike for relaxation oscillations). Therefore, the LCO can be characterized theoretically only from the two-dimensional slow subsystem (40). This situation also corresponds to a modulated response, in terms of amplitude and phase, 


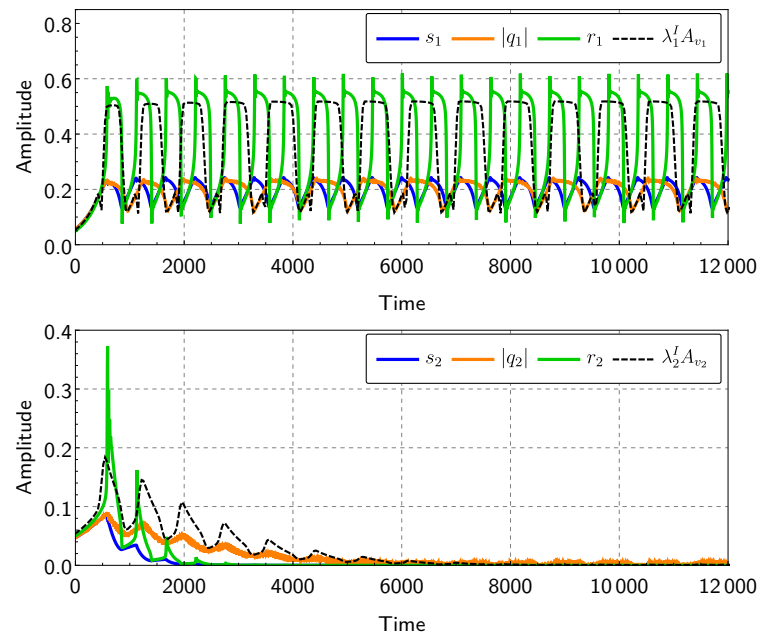

(a)

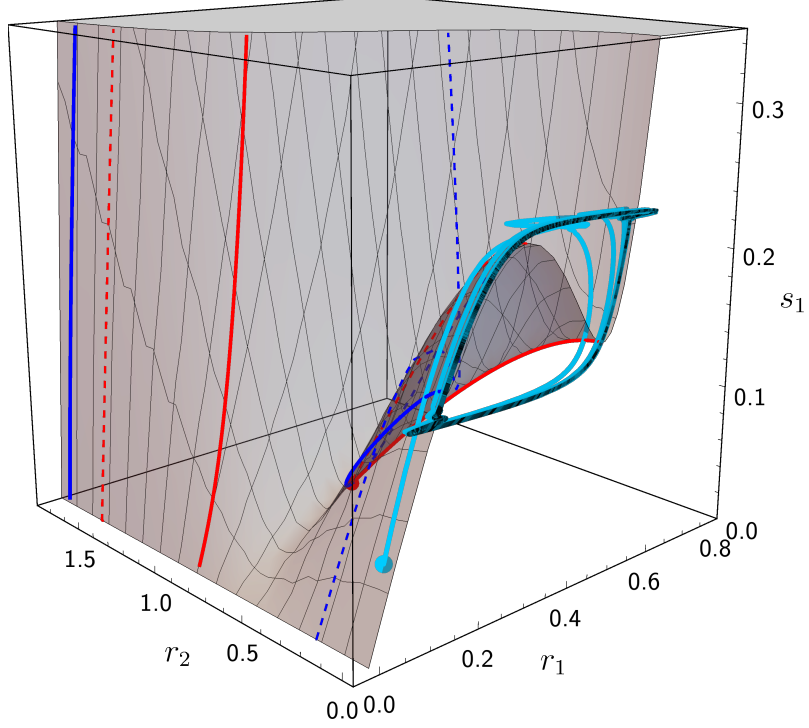

(c)

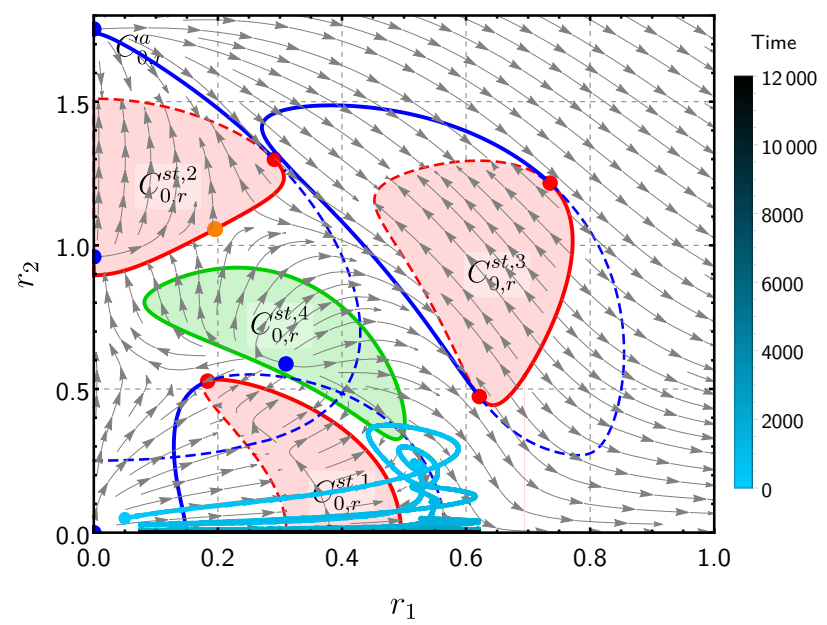

(b)

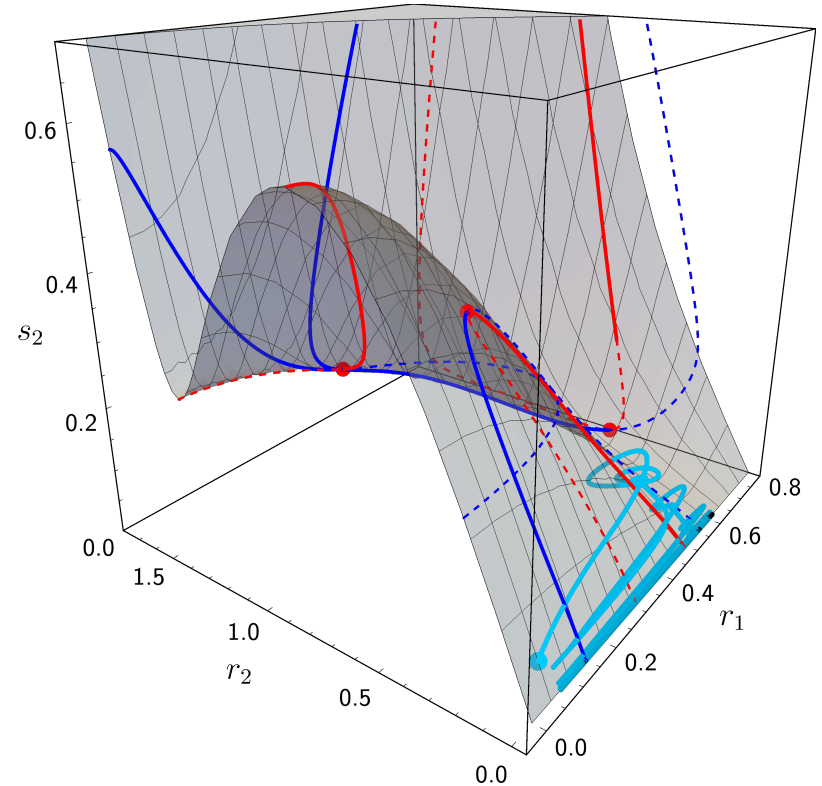

(d)

Figure 8: Competition between two kinds of sustained relaxation oscillations ( $1^{\text {st }}$ example). Same caption as in Fig. 7 with the initial condition $\mathbf{r}_{0,5}=(0.05,0.05)$. The set of parameters (58) is used.

of the mechanical system (13), but here due to a limit cycle of the slow flow instead of relaxation oscillations of the slow flow as in the SMR case.

Finally, once again, the predictions obtained with the slow flow (19) are in good agreement with that of the complete system (12) (see Figs. 10(a) and 10(c)), which allows us to validate the asymptotic analysis presented in Sect. 3.

\section{Conclusion}

The possibility of mitigating simultaneously two unstable modes of a linear MDOF primary system using a nonlinear energy sink (NES) has been investigated in this paper by means of an asymptotic analysis 


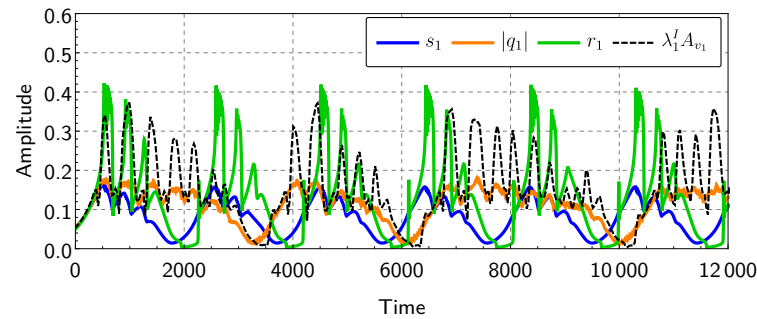

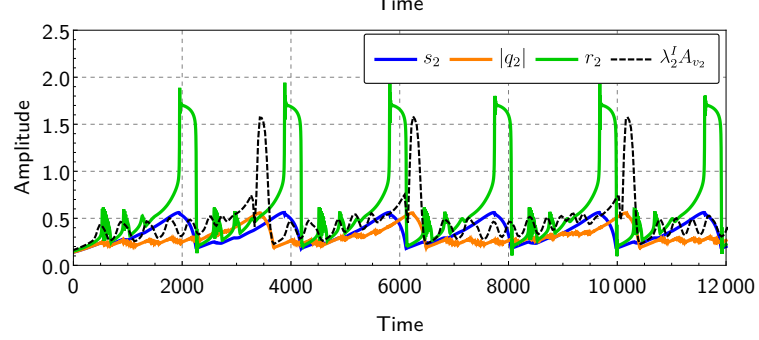

(a)

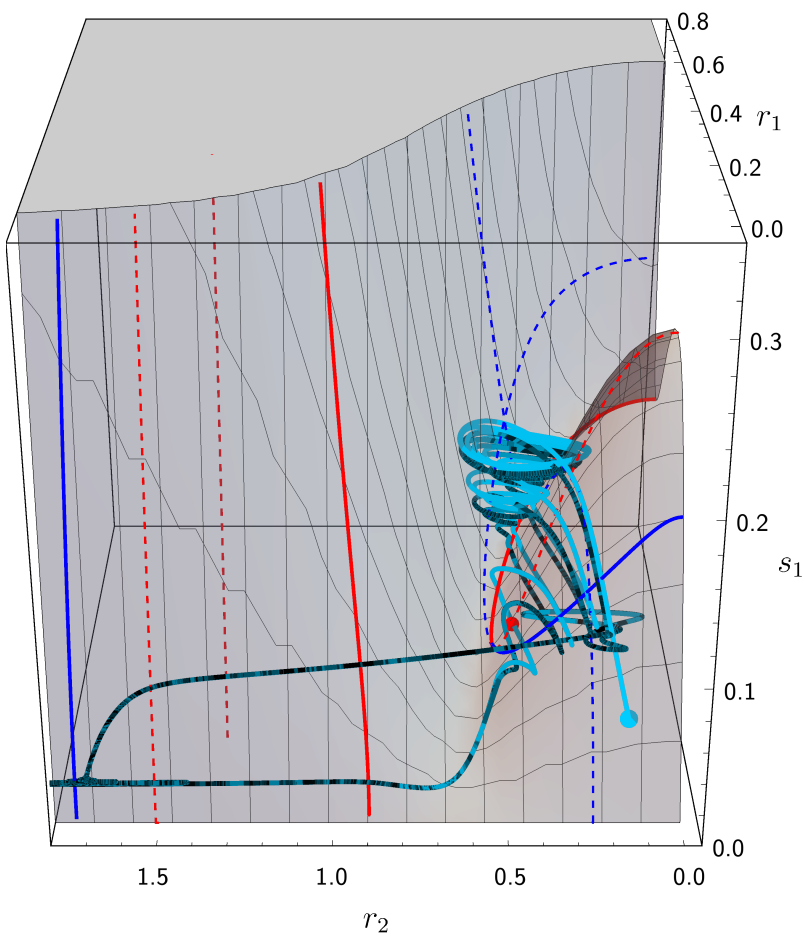

(c)

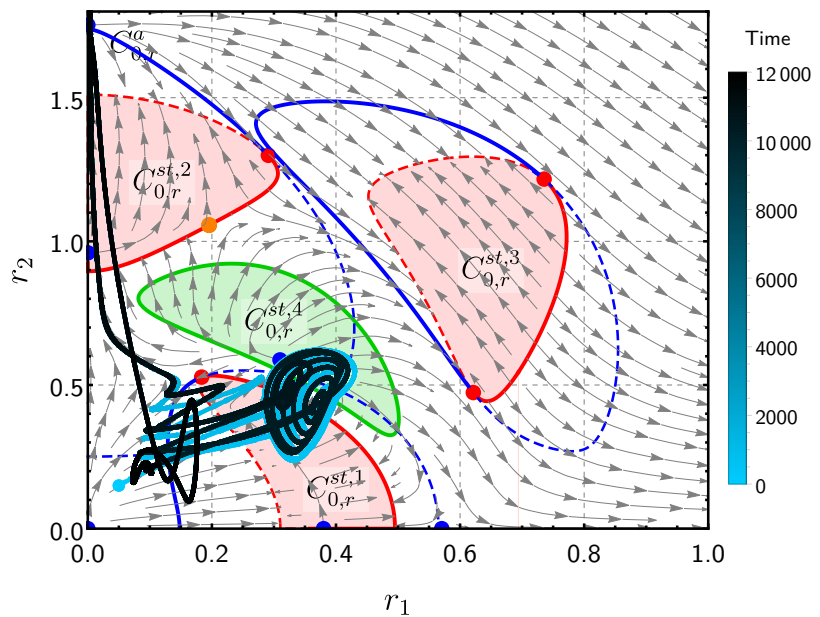

(b)

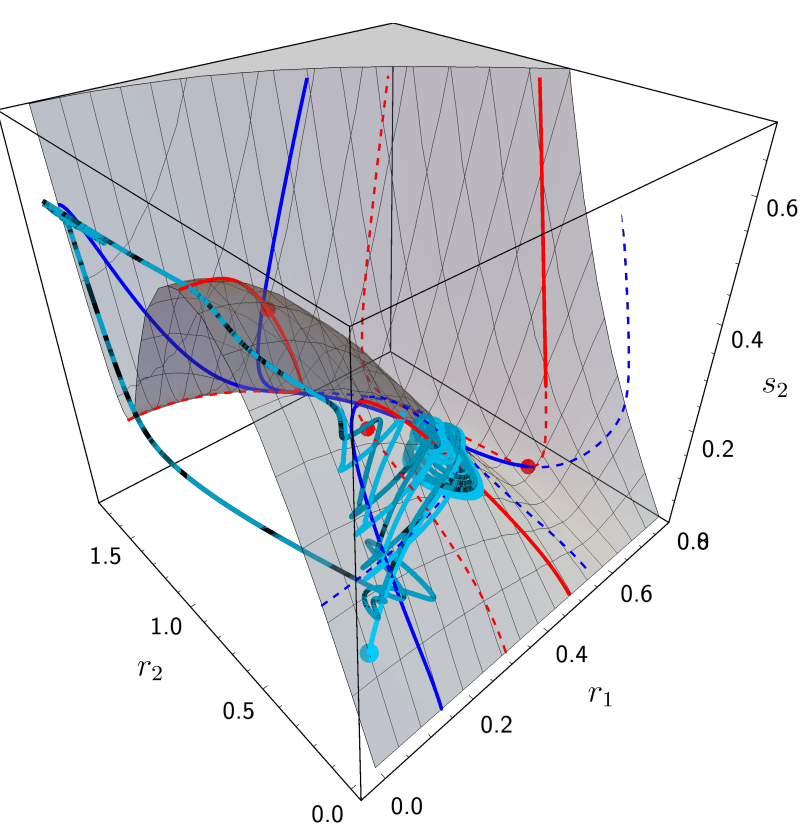

(d)

Figure 9: Competition between two kinds of sustained relaxation oscillations (2 ${ }^{\text {nd }}$ example). Same caption as in Fig. 7 with the initial condition $\mathbf{r}_{0,6}=(0.05,0.15)$. The set of parameters $(58)$ is used.

complemented by numerical simulations. This study focuses on a 4-DOF phenomenological friction system having two unstable modes and coupled to a single NES.

The asymptotic analysis begins with the well-known biorthogonal transformation to diagonalize the primary system for the purpose of reducing its dynamics only to the two unstable modes. A complexificationaveraging process is applied at the vicinity of the two 1:1-1:1 resonances at the natural frequencies of the unstable modes, leading to the slow flow of the system which takes the form of a $(4,2)$-fast-slow system. The 


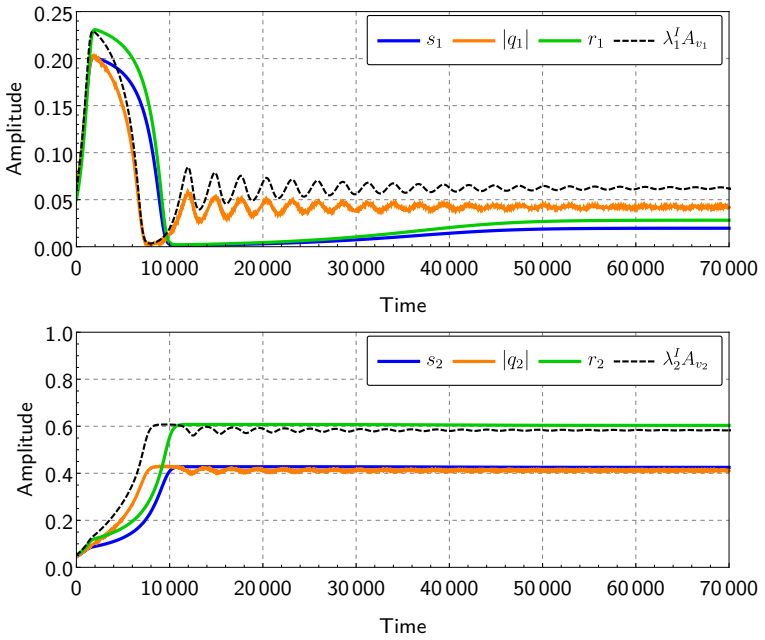

(a)
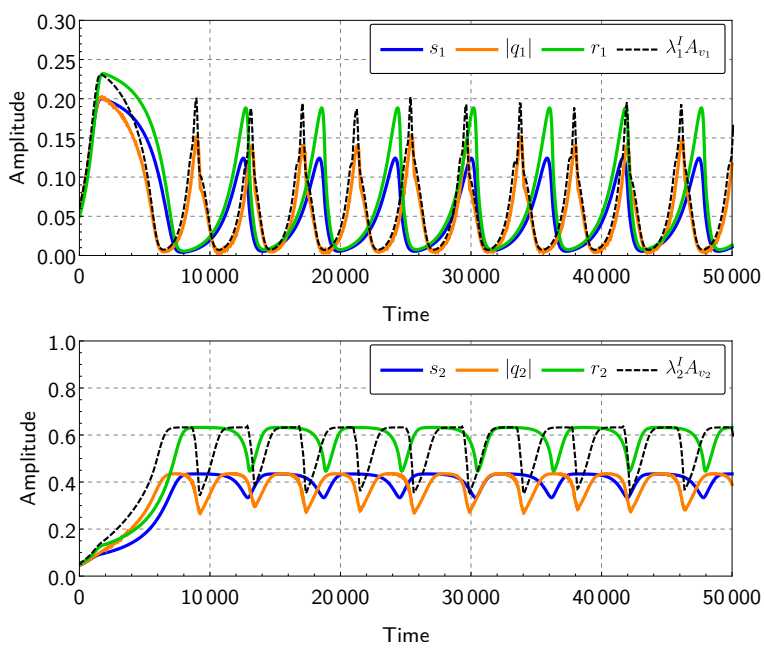

(c)

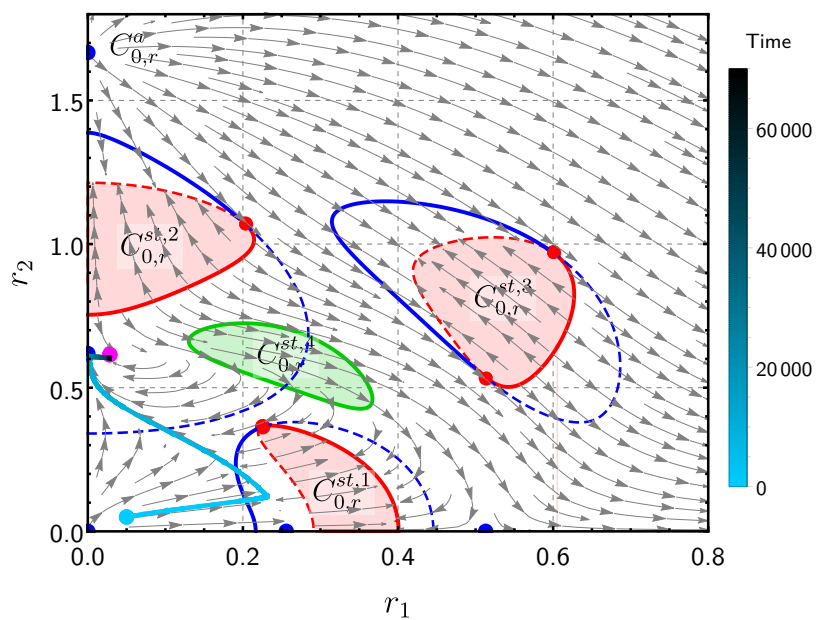

(b)

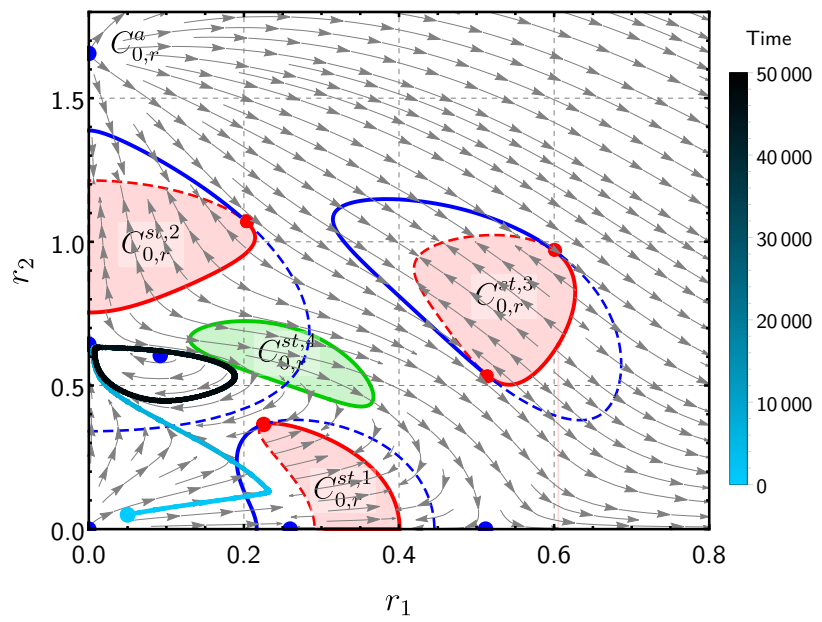

(d)

Figure 10: Bifurcation of a fixed point to a limit cycle of the slow subsystem. Same caption as in Fig. 4 with the initial condition $\mathbf{r}_{0,7}=(0.05,0.05)$. The parameters (59) are used, with (a) and (b) $\gamma=0.09053$ and (c) and (d) $\gamma=0.09075$.

slow variables characterize the two unstable modes of the primary system whereas the four fast variables describe the relative motion between the NES and the primary system (amplitudes and phases of the two frequency components relative to the two unstable modes). The slow flow is finally analyzed by means of the geometric singular perturbation theory. The critical manifold $C_{0}$ is obtained and, contrary to the case of a single unstable mode, it consists in a 2-dimensional parametric surface with respect to the two fast variables $r_{1}$ and $r_{2}$ which evolve in the whole 6 -dimensional variable space, and which characterize the frequency components of the NES dynamics with respect to the two unstable modes. The critical manifold appears as the union of a normally hyperbolic attracting domain and four normally hyperbolic saddle type domains (with finite dimensions). Two of them meet the attracting part at fold curves and it can be shown by analyzing, inter alia, the transversality condition, that these fold curves can be split into successive incoming and outgoing sections, enabling jumps and complex motions of the slow flow, such as successive slow and fast epochs (i.e. relaxation oscillations). Some relaxation oscillation scenarios have been deduced from the asymptotic analysis, among which simple relaxation oscillations around one of the normally hyperbolic 
saddle type domains, or multi-relaxation oscillations around two of the normally hyperbolic saddle type domains. These relaxation oscillation scenarios and the stable fixed points of the slow flow define possible sustained mitigated regimes of the physical system such as strongly modulated responses (SMR), complex SMR, periodic or quasi-periodic regimes.

Direct numerical integrations of the system have been performed. First of all, the simulations validated that it was possible to mitigate simultaneously two unstable modes by means of a single NES. The two unstable modes can be completely eliminated, or mitigated through one of the sustained regimes (periodic, quasi-periodic, SMR). Secondly, the simulations allowed us to study the notion of basin of attraction. Indeed, because of the two-dimensional nature of $C_{0}$ and the fact that, for a given set of parameters, the system may have several stable attractors (stable fixed points or sustained relaxation oscillations) this notion has to be considered. Three kinds of competitions have been observed, respectively between two stable fixed points of the slow flow, between a sustained relaxation oscillation and a stable fixed point of the slow flow and between two kinds of sustained relaxation oscillations. In each situation considered, a good agreement has been observed between the theoretical results and the numerical simulations, which validates the proposed asymptotic analysis. Moreover, the numerical simulations highlighted that the two unstable modes could also be mitigated through a strongly modulated response defined from a limit cycle of the slow system at the slow time scale.

As a general conclusion, when it is coupled to a linear system which has two unstable modes, a single NES is able to produce bounded motions of various kinds, thoroughly studied in this paper. In real life, unstable mechanical systems are in general nonlinear and therefore they undergo limit cycle oscillations which can be dangerous if they are of significant amplitude. As a perspective, from an engineering point of view, the NES may be designed to produce effective bounded motions, i.e. with a lower amplitude than that of the system without the NES. The present study might constitute a tool to perform this design. The amplitude of the oscillations of the resulting bounded motions may also be compared with those provided by other mitigation methods.

\section{Acknowledgements}

We thank the anonymous reviewers for their careful reading of our manuscript and their many relevant comments and suggestions.

\section{Appendix A. Coefficients of the slow-fow system (19)}

The coefficients $a_{i}$ for $i=1, \ldots, 10$, characterizing the functions $\mathbf{f}$ and $\mathbf{g}$ which define the slow-fow system (19), are given by

$$
\begin{gathered}
a_{1}=\frac{\eta_{a} L_{71}^{\mathrm{I}} R_{51}^{I}}{\sigma}-\eta_{a} L_{51}^{I} R_{51}^{I}+\frac{\Omega_{a}^{2} L_{71}^{I} R_{11}^{I}}{\sigma}-\Omega_{a}^{2} L_{51}^{I} R_{11}^{I}-\frac{R_{51}^{R} \eta_{a} L_{71}^{R}}{\sigma}+R_{51}^{R} \eta_{a} L_{51}^{R}-\frac{R_{11}^{R} \Omega_{a}^{2} L_{71}^{R}}{\sigma}+ \\
R_{11}^{R} \Omega_{a}^{2} L_{51}^{R}+\gamma L_{61}^{I} R_{11}^{I}-\eta_{1} L_{51}^{I} R_{51}^{I}-L_{51}^{I} R_{11}^{I}-\gamma R_{11}^{R} L_{61}^{R}+\eta_{1} R_{51}^{R} L_{51}^{R}+R_{11}^{R} L_{51}^{R}+\rho_{1}, \\
a_{2}=\frac{\Omega_{a}^{2} L_{71}^{I}}{2 \sigma \lambda_{1}^{I}}-\frac{\Omega_{a}^{2} L_{51}^{I}}{2 \lambda_{1}^{I}}+\frac{\eta_{a} L_{71}^{R}}{2 \sigma}-\frac{1}{2} \eta_{a} L_{51}^{R}+\frac{\gamma L_{61}^{I}}{2 \lambda_{1}^{-I}}-\frac{L_{51}^{I}}{2 \lambda_{1}^{I}}-\frac{1}{2} \eta_{1} L_{51}^{R}, \\
a_{3}=\frac{\eta_{a} L_{71}^{I}}{2 \sigma}-\frac{1}{2} \eta_{a} L_{51}^{I}-\frac{\Omega_{a}^{2} L_{71}^{R}}{2 \sigma \lambda_{1}^{I}}+\frac{\Omega_{a}^{2} L_{51}^{R}}{2 \lambda_{1}^{I}}-\frac{1}{2} \eta_{1} L_{51}^{I}-\frac{\gamma L_{61}^{R}}{2 \lambda_{1}^{I}}+\frac{L_{51}^{R}}{2 \lambda_{1}^{I}}, \\
a_{4}=\frac{\eta_{a} L_{73}^{I} R_{53}^{I}}{\sigma}-\eta_{a} L_{53}^{I} R_{53}^{I}+\frac{\Omega_{a}^{2} L_{73}^{I} R_{13}^{I}}{\sigma}-\Omega_{a}^{2} L_{53}^{I} R_{13}^{I}-\frac{R_{53}^{R} \eta_{a} L_{73}^{R}}{\sigma}+R_{53}^{R} \eta_{a} L_{53}^{R}-\frac{R_{13}^{R} \Omega_{a}^{2} L_{73}^{R}}{\sigma}+ \\
R_{13}^{R} \Omega_{a}^{2} L_{53}^{R}+\gamma L_{63}^{I} R_{13}^{I}-\eta_{1} L_{53}^{I} R_{53}^{I}-L_{53}^{I} R_{13}^{I}-\gamma R_{13}^{R} L_{63}^{R}+\eta_{1} R_{53}^{R} L_{53}^{R}+R_{13}^{R} L_{53}^{R}+\rho_{2},
\end{gathered}
$$




$$
\begin{gathered}
a_{5}=\frac{\Omega_{a}^{2} L_{73}^{I}}{2 \sigma \lambda_{2}^{I}}-\frac{\Omega_{a}^{2} L_{53}^{I}}{2 \lambda_{2}^{I}}+\frac{\eta_{a} L_{73}^{R}}{2 \sigma}-\frac{1}{2} \eta_{a} L_{53}^{R}+\frac{\gamma L_{63}^{I}}{2 \lambda_{2}^{I}}-\frac{L_{53}^{I}-\frac{1}{2} \eta_{1} L_{53}^{R}}{2 \lambda_{2}^{I}}, \\
a_{6}=\frac{\eta_{a} L_{73}^{I}}{2 \sigma}-\frac{1}{2} \eta_{a} L_{53}^{I}-\frac{\Omega_{a}^{2} L_{73}^{R}}{2 \sigma \lambda_{2}^{I}}+\frac{\Omega_{a}^{2} L_{53}^{R}}{2 \lambda_{2}^{I}}-\frac{1}{2} \eta_{1} L_{53}^{I}-\frac{\gamma L_{63}^{R}}{2 \lambda_{2}^{I}}+\frac{\lambda_{2}^{I} L_{53}^{R} L_{53}^{R}}{2 \lambda_{2}^{I} L_{53}^{R}}, \\
a_{7}=-R_{51}^{R} \eta_{a}+R_{71}^{R} \eta_{a}-R_{11}^{R} \Omega_{a}^{2}+R_{31}^{R} \Omega_{a}^{2}-\gamma R_{21}^{R} \Omega_{2}^{2}-\eta_{1} R_{51}^{R}-R_{11}^{R} \\
a_{8}=\eta_{a} R_{51}^{I}-\eta_{a} R_{71}^{I}+\Omega_{a}^{2} R_{11}^{I}-\Omega_{a}^{2} R_{31}^{I}+\gamma \Omega_{2}^{2} R_{21}^{I}+\eta_{1} R_{51}^{I}+R_{11}^{I} \\
a_{9}=-R_{53}^{R} \eta_{a}+R_{73}^{R} \eta_{a}-R_{13}^{R} \Omega_{a}^{2}+R_{33}^{R} \Omega_{a}^{2}-\gamma R_{23}^{R} \Omega_{2}^{2}-\eta_{1} R_{53}^{R}-R_{13}^{R}
\end{gathered}
$$

and

$$
a_{10}=\eta_{a} R_{53}^{I}-\eta_{a} R_{73}^{I}+\Omega_{a}^{2} R_{13}^{I}-\Omega_{a}^{2} R_{33}^{I}+\gamma \Omega_{2}^{2} R_{23}^{I}+\eta_{1} R_{53}^{I}+R_{13}^{I}
$$

\section{References}

[1] A. F. Vakatis, O. Gendelman, L. a. Bergman, D. M. McFarland, G. Kerschen, Y. Lee, Nonlinear Targeted Energy Transfer in Mechanical and Structural Systems, no. 156, Springer, 2009. doi:10.1007/978-1-4020-9130-8.

[2] Z. Lu, Z. Wang, Y. Zhou, X. Lu, Nonlinear dissipative devices in structural vibration control: A review, Journal of Sound and Vibration 423 (2018) 18-49.

[3] Y. S. Lee, A. F. Vakakis, L. A. Bergman, D. M. McFarland, Suppression of limit cycle oscillations in the van der Pol oscillator by means of passive non-linear energy sinks, Structural Control and Health Monitoring 13 (1) (2006) 41-75. doi:10.1002/stc.143.

[4] O. V. Gendelman, T. Bar, Bifurcations of self-excitation regimes in a Van der Pol oscillator with a nonlinear energy sink, Physica D 239 (3-4) (2010) 220-229. doi:10.1016/j.physd.2009.10.020.

[5] E. Domany, O. Gendelman, Dynamic responses and mitigation of limit cycle oscillations in van der pol-duffing oscillator with nonlinear energy sink, Journal of Sound and Vibration 332 (2013) 5489-5507.

[6] Y. S. Lee, A. F. Vakakis, L. A. Bergman, D. M. McFarland, G. Kerschen, Suppression aeroelastic instability using broadband passive targeted energy transfers, part 1: Theory, AIAA Journal 45 (3) (2007) 693-711. doi:10.2514/1.24062.

[7] Y. S. Lee, A. F. Vakakis, L. A. Bergman, D. M. McFarland, G. Kerschen, Suppression aeroelastic instability using broadband passive targeted energy transfers, part 2: Experiments, AIAA Journal 45 (3) (2007) 2391-2400. doi:10.2514/ 1.24062.

[8] Y. S. Lee, A. F. Vakakis, D. M. Bergman, L. A. McFarland, G. Kerschen, Enhancing the robustness of aeroelastic instability suppression using multi-degree-of-freedom nonlinear energy sinks, AIAA Journal 46 (6) (2008) 1371-1394.

[9] O. Gendelman, A. Vakakis, L. Bergman, D. McFarland, Asymptotic analysis of passive nonlinear suppression of aeroelastic instabilities of a rigid wing in subsonic flow, SIAM Journal on Applied Mathematics 70 (5) (2010) 1655-1677. doi: 10.1137/090754819.

[10] B. Vaurigaud, L. Manevitch, C.-H. Lamarque, Passive control of aeroelastic instability in a long span bridge model prone to couple dflutter using targeted energy transfer, Journal of Sound and Vibration 330 (2011) 2580-2595.

[11] H.-l. Guo, Y.-s. Chen, T.-z. Yang, Limit cycle oscillation suppression of 2-dof airfoil using nonlinear energy sink, Appl. Math. Mech. -Engl. Ed. 34 (10) (2013) 1277-1290.

[12] A. Luongo, D. Zulli, Aeroelastic instability analysis of nes-controlled systems via a mixed multiple scale/harmonic balance method, Journal of Vibration and Control 20 (13) (2014) 1985-1998. arXiv:http://jvc.sagepub.com/content/early/ 2013/06/17/1077546313480542.full.pdf+html, doi:10.1177/1077546313480542. URL http://jvc.sagepub.com/content/early/2013/06/17/1077546313480542.abstract

[13] D. R. Q. Pacheco, F. D. Marques, A. J. M. Ferreira, D. R. Q. Pacheco, F. D. Marques, Panel flutter suppression with nonlinear energy sinks: numerical modeling and analysis, International Journal of Non-Linear Mechanics 106 (2018) $108-114$.

[14] R. K. R. Tumkur, E. Domany, O. V. Gendelman, A. Masud, L. A. Bergman, A. F. Vakakis, Reduced-order model for laminar vortex-induced vibration of a rigid circular cylinder with an internal nonlinear absorber, Communications in Nonlinear Science and Numerical Simulation 18 (7) (2013) 1916-1930. doi:10.1016/j.cnsns.2012.11.028. URL http://dx.doi.org/10.1016/j.cnsns.2012.11.028

[15] H. L. Dai, A. Abdelkefi, L. Wang, Vortex-induced vibrations mitigation through a nonlinear energy sink, Communications in Nonlinear Science and Numerical Simulation 42 (2017) 22-36. doi:10.1016/j.cnsns.2016.05.014. 
[16] B. Bergeot, S. Bellizzi, B. Cochelin, Analysis of steady-state response regimes of a helicopter ground resonance model including a non-linear energy sink attachment, International Journal of Non-Linear Mechanics 78 (2016) 72 - 89. doi: http://dx.doi.org/10.1016/j.ijnonlinmec.2015.10.006.

URL http://www.sciencedirect.com/science/article/pii/S0020746215002000

[17] B. Bergeot, S. Bellizzi, B. Cochelin, Passive suppression of helicopter ground resonance using nonlinear energy sinks attached on the helicopter blades, Journal of Sound and Vibration 392 (2017) 41-55. doi:10.1016/j.jsv.2016.12.039.

[18] E. Gourc, S. Seguy, G. Michon, A. Berlioz, Chatter Control in Turning Process with a Nonlinear Energy Sink, Advanced Materials Research 698 (2013) 89-98. doi:10.4028/www.scientific.net/AMR.698.89.

[19] A. Nankali, Y. S. Lee, T. Kalmar-Nagy, Targeted energy transfers for suppressing regenerative machine tool vibrations, Journal of Computational and Nonlinear Dynamics Transactions of the ASME 12 (2017) 011010-1-011010-11.

[20] B. Bergeot, S. Berger, S. Bellizzi, Mode coupling instability mitigation in friction systems by means of nonlinear energy sinks : numerical highlighting and local stability analysis, Journal of Vibration and Control 24 (15) (2017) $3487-3511$. doi: $10.1177 / 1077546317707101$.

[21] B. Bergeot, S. Bellizzi, Asymptotic analysis of passive mitigation of dynamic instability using a nonlinear energy sink network, Nonlinear Dynamics 94 (2) (2018) 1501-1522. doi:10.1007/s11071-018-4438-0. URL http://link.springer.com/10.1007/s11071-018-4438-0

[22] B. Bergeot, S. Bellizzi, Steady-state regimes prediction of a multi-degree-of-freedom unstable dynamical system coupled to a set of nonlinear energy sinks, Mechanical Systems and Signal Processing 131 (2019) 728-750.

[23] T. T. Pham, C. H. Lamarque, A. T. Savadkoohi, Multi-resonance capturing in a two-degree-of-freedom system under two different harmonic excitations, Journal of Vibration and Control 18 (3) (2012) 451-466. doi:10.1177/1077546311404268.

[24] T. T. Pham, C. H. Lamarque, S. Pernot, Passive control of one degree of freedom nonlinear quadratic oscillator under combination resonance, Communications in Nonlinear Science and Numerical Simulation 16 (5) (2011) 2279-2288. doi: $10.1016 / j$.cnsns.2010.04.050. URL http://dx.doi.org/10.1016/j.cnsns.2010.04.050

[25] S. Bellizzi, R. Côte, M. Pachebat, Responses of a two degree-of-freedom system coupled to a nonlinear damper under multi-forcing frequencies, Journal of Sound and Vibration 332 (2013) 1639-1653.

[26] R. Côte, M. Pachebat, S. Bellizzi, Experimental evidence of simultaneous multi-resonance noise reduction using an absorber with essential nonlinearity under two excitation frequencies, Journal of Sound and Vibration 333 (2014) 5057-5076.

[27] G. Kerschen, Y. S. Lee, A. F. Vakakis, D. M. McFarland, L. A. Bergman, Irreversible Passive Energy Transfer in Coupled Oscillators with Essential Nonlinearity, SIAM Journal on Applied Mathematics 66 (2) (2005) 648-679. doi:10.1137/ 040613706.

URL http://epubs.siam.org/doi/abs/10.1137/040613706

[28] A. F. Vakakis, L. I. Manevitch, O. Gendelman, L. Bergman, Dynamics of linear discrete systems connected to local, essentially non-linear attachments, Journal of Sound and Vibration 264 (3) (2003) 559-577. doi:10.1016/S0022-460X (02) 01207-5.

[29] A. F. Vakakis, D. M. McFarland, L. Bergman, L. I. Manevitch, O. Gendelman, Isolated resonance captures and resonance capture cascades leading to single- or multi-mode passive energy pumping in damped coupled oscillators, Journal of Vibration and Acoustics 126 (2) (2004) 235-244. doi:10.1115/1.1687397.

[30] G. Habib, F. Romeo, The tuned bistable nonlinear energy sink, Nonlinear Dynamics 89 (2017) $179-196$.

[31] K. Foroutan, A. Jalali, H. Ahmadi, Investigations of energy absorption using tuned bistable nonlinear energy sink with local and global potentials, Journal of Sound and Vibration 447 (2019) 155-169.

[32] E. Denimal, L. Nechak, J.-J. Sinou, S. Nacivet, Kriging surrogate models for predicting the complex eigenvalues of mechanical systems subjected to friction-induced vibration, Shock and Vibration Hindawi Publishing Corporation ID 3586230 (2016) 22 pages.

[33] E. Denimal, L. Nechak, J.-J. Sinou, S. Nacivet, A novel hybrid surrogate model and its application on a mechanical system subjected to friction-induced vibration, Journal of Sound and Vibration 434 (2018) 456-474.

[34] L. I. Manevitch, Complex representation of dynamics of coupled nonlinear oscillators, in: L. Uvarova, A. Arinstein, A. Latyshev (Eds.), Mathematical Models of Non-Linear Excitations, Transfer, Dynamics, and Control in Condensed Systems and Other Media, Springer US, 1999, pp. 269-300. doi:10.1007/978-1-4615-4799-0\_24.

[35] Y. Starosvetsky, O. V. Gendelman, Strongly modulated response in forced 2dof oscillatory system with essential mass and potential asymmetry, Physica D 237 (13) (2008) 1719-1733. doi:10.1016/j.physd.2008.01.019.

[36] C. Kuehn, Multiple Time Scale Dynamics, 1st Edition, Vol. 191 of Applied Mathematical Sciences, Springer International Publishing, 2015.

[37] E. Benoît, J. Callot, F. Diener, M. Diener, Chasse au canard ("duck hunting"), Collectanea Mathematica 32 (1-2) (1981) 37-119. 